https://helda.helsinki.fi

\title{
EFFICIENT COUNTING WITH OPTIMAL RESILIENCE
}

\section{Lenzen, Christoph}

2017

Lenzen , C , Rybicki , J \& Suomela , J 2017 , ' EFFICIENT COUNTING WITH OPTIMAL

RESILIENCE ' , SIAM Journal on Computing , vol. 46 , no. 4 , pp. 1473-1500 . https://doi.org/10.1137/16M107877X

http://hdl.handle.net/10138/224608

https://doi.org/10.1137/16M107877X

publishedVersion

Downloaded from Helda, University of Helsinki institutional repository.

This is an electronic reprint of the original article.

This reprint may differ from the original in pagination and typographic detail.

Please cite the original version. 


\title{
EFFICIENT COUNTING WITH OPTIMAL RESILIENCE*
}

\author{
CHRISTOPH LENZEN ${ }^{\dagger}$, JOEL RYBICKI ${ }^{\dagger} \S$, AND JUKKA SUOMELA ${ }^{\S}$
}

\begin{abstract}
Consider a complete communication network of $n$ nodes, where the nodes receive a common clock pulse. We study the synchronous $c$-counting problem: given any starting state and up to $f$ faulty nodes with arbitrary behavior, the task is to eventually have all correct nodes labeling the pulses with increasing values modulo $c$ in agreement. Thus, we are considering algorithms that are self-stabilizing despite Byzantine failures. In this work, we give new algorithms for the synchronous counting problem that (1) are deterministic, (2) have optimal resilience, (3) have a linear stabilization time in $f$ (asymptotically optimal), (4) use a small number of states, and, consequently, (5) communicate a small number of bits per round. Prior algorithms either resort to randomization, use a large number of states and need high communication bandwidth, or have suboptimal resilience. In particular, we achieve an exponential improvement in both state complexity and message size for deterministic algorithms. Moreover, we present two complementary approaches for reducing the number of bits communicated during and after stabilization.
\end{abstract}

Key words. self-stabilization, Byzantine fault-tolerance

AMS subject classifications. 68M14, 68M15, 68Q25, 68W15

DOI. $10.1137 / 16 \mathrm{M} 107877 \mathrm{X}$

1. Introduction. In this work, we design space- and communication-efficient, selfstabilizing, Byzantine fault-tolerant algorithms for the synchronous counting problem. We are given a complete communication network on $n$ nodes with arbitrary initial states. There are up to $f$ faulty nodes. The task is to synchronize the nodes so that all nonfaulty nodes will count rounds modulo $c$ in agreement. For example, here is a possible execution for $n=4$ nodes, $f=1$ faulty node, and counting modulo $c=3$; the execution stabilizes after $t=5$ rounds:

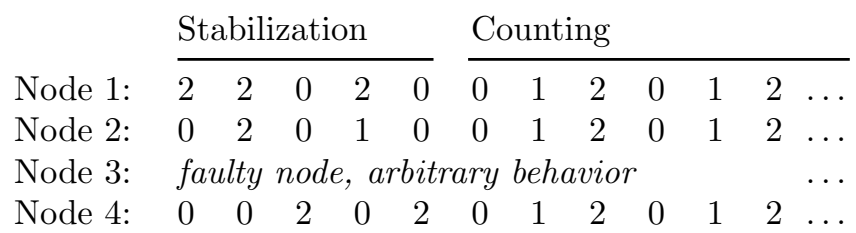

Synchronous counting is a coordination primitive that can be used, e.g., in large integrated circuits to synchronize subsystems to easily implement mutual exclusion and time division multiple access in a fault-tolerant manner. Note that in this context, it is natural to assume that a synchronous clock signal is available, but the clocking system usually does not provide explicit round numbers. Solving synchronous counting thus yields highly dependable round counters for subcircuits.

${ }^{*}$ Received by the editors June 8, 2016; accepted for publication (in revised form) May 15, 2017; published electronically August 22, 2017. This paper is an extended and revised version of two preliminary conference reports $[24,26]$ that appeared in the Proceedings of the 34th Annual ACM Symposium on Principles of Distributed Computing (PODC 2015) and in the Proceedings of the 29th International Symposium on Distributed Computing (DISC 2015).

http://www.siam.org/journals/sicomp/46-4/M107877.html

${ }^{\dagger}$ Department of Algorithms and Complexity, Max Planck Institute for Informatics, Saarland Informatics Campus, 66123 Saarbrucken, Germany (clenzen@mpi-inf.mpg.de).

${ }^{\ddagger}$ Department of Algorithms and Complexity, Max Planck Institute for Informatics, Saarland Informatics Campus, 66123 Saarbrucken, Germany. Current address: Department of Biosciences, FI-00014 University of Helsinki, Helsinki, Finland (joel.rybicki@helsinki.fi).

$\S$ Helsinki Institute for Information Technology HIIT, and Department of Computer Science, Aalto University, FI-02150 Espoo, Finland (jukka.suomela@aalto.fi). 
If we neglect communication, counting and consensus are essentially equivalent $[13$, 14, 15]. In particular, many lower bounds on (binary) consensus directly apply to the counting problem $[16,20,27]$. However, the known generic reduction of counting to consensus incurs a factor- $f$ overhead in space and message size. In this work, we present techniques that reduce the number of bits nodes broadcast in each round to $O\left(\log ^{2} f+\log c\right)$.

1.1. Contributions. Our contributions constitute of two parts. First, we give novel space-efficient deterministic algorithms for synchronous counting with optimal resilience and fast stabilization time. Second, we show how to extend these algorithms in a way that reduces the number of communicated bits during and after stabilization.

Space-efficient counting algorithms. In this work, we take the following approach for devising communication-efficient counting algorithms: we first design space-efficient algorithms, that is, algorithms in which each node stores only a few bits between consecutive rounds. Space-efficient algorithms are particularly attractive from the perspective of fault-tolerant systems: if we can keep the number of state bits small, we can also reduce the overall complexity of the system, which in turn makes it easier to use highly reliable components for an implementation.

Once we have algorithms that need only a small number of bits to encode the local state of a node, we also get algorithms that use small messages: the nodes can simply broadcast their entire state to everyone. Our main result is summarized in the following theorem; here $f$-resilient means that we can tolerate up to $f$ faulty nodes.

TheOREM 1.1. For any integers $c, n>1$ and $f<n / 3$, there exists a deterministic $f$-resilient synchronous c-counter that runs on $n$ nodes, stabilizes in $O(f)$ rounds, and uses $O\left(\log ^{2} f+\log c\right)$ bits to encode the state of a node.

Our main technical contribution is a recursive construction that shows how to "amplify" the resilience of a synchronous counting algorithm. Given a synchronous counter for some values of $n$ and $f$, we will show how to design synchronous counters for larger values of $n$ and $f$ with a very small increase in time and state complexity. This has two direct applications:

1. From a practical perspective, we can apply existing computer-designed algorithms (e.g., $n=4$ and $f=1$ ) as a building block in order to design efficient deterministic algorithms for a moderate number of nodes (e.g., $n=36$ and $f=7)$.

2. From a theoretical perspective, we can design deterministic algorithms for synchronous counting for any $n$ and any $f<n / 3$, with a stabilization time of $\Theta(f)$, and with only $O\left(\log ^{2} f\right)$ bits of state per node.

The state complexity and message size is an exponential improvement over prior work, and the stabilization time is asymptotically optimal for deterministic algorithms [20].

Reducing communication after stabilization. In our deterministic algorithms, each node need only store a few number of bits between consecutive rounds, and thus, a node can, e.g., afford to broadcast its entire state to all other nodes in each round. Moreover, we present a technique to reduce the number of communicated bits further.

We give a deterministic construction in which after stabilization each node broadcasts $O(1+B \log B)$ bits every $\kappa$ rounds, where $B=O(\log c / \log \kappa)$, for an essentially unconstrained choice of $\kappa$, at the expense of additively increasing the stabilization time by $O(\kappa)$. In particular, for the special case of optimal resilience and polynomial counter size, we obtain the following result. 
Corollary 1.2. For any $n>1$ and $c=n^{O(1)}$ that is an integer multiple of $n$, there exists a synchronous c-counter that runs on $n$ nodes, has optimal resilience $f=\lfloor(n-1) / 3\rfloor$, stabilizes in $\Theta(n)$ rounds, requires $O\left(\log ^{2} n\right)$ bits to encode the state of a node, and for which after stabilization correct nodes broadcast aysmptotically optimal $O(1)$ bits per $\Theta(n)$ rounds.

We remark that in the above result we simply reduce the frequency of communication and the size of messages instead of, e.g., bounding the number of nodes communicating in any given round (known as broadcast efficiency) [28]. In our work, we exploit synchrony after stabilization to schedule communication, and thus, our approach is to be contrasted with attempting to reduce the total number of communication partners or communicating nodes after stabilization [9, 10, 28].

Reducing the number of messages. To substantiate the conjecture that finding algorithms with small state complexity may lead to highly communication-efficient solutions, we proceed to consider a slightly stronger synchronous pulling model. In this model, a node may send a request to another node and receive a response in a single round, based on the state of the responding node at the beginning of the round. The cost for the exchange is then attributed to the pulling node; in a circuit, this translates to each node being assigned an energy budget that it uses to "pay" for the communication it triggers. In this model, it is straightforward to combine our recursive construction used in Theorem 1.1 with random sampling to obtain the following results:

1. We can achieve the same asymptotic running time and state complexity as the deterministic algorithm from Theorem 1.1 with each node pulling only polylog $n$ messages in each round. The price is that the resulting algorithm retains a probability of $n^{-\operatorname{polylog} n}$ to fail in each round even after stabilization and that the resilience is $f<n /(3+\gamma)$ for any constant $\gamma>0$.

2. If the failing nodes are chosen independently of the algorithm, we can fix the random choices. This results in a pseudorandom algorithm which stabilizes with a probability of $1-n^{- \text {polylog } n}$ and in this case keeps counting correctly.

1.2. Our approach. Most prior deterministic algorithms for synchronous counting and closely related problems utilize consensus protocols [14, 22]. Indeed, if we ignore space and communication, reductions exist both ways showing that the problems are more or less equivalent [12]; see section 2 for further discussion on prior work.

However, to construct fast space- and communication-efficient counters, we are facing a chicken-and-egg problem:

- From counters to consensus: If the correct nodes could agree on a counter, they could jointly run a single instance of synchronous consensus.

- From consensus to counters: If the nodes could run a consensus algorithm, they could agree on a counter.

A key step to circumvent this obstacle is the following observation:

- From unreliable counters to consensus: If the correct nodes can agree on a counter at least for a while, they can jointly run a single instance of consensus.

- From consensus to reliable counters: Consensus can be then used to facilitate agreement on the output counter, and it is possible to maintain agreement even if the underlying unreliable counters fail later on.

The task of constructing counters that are correct only once in a while is easier; in particular, it does not require that we solve consensus in the process. As our main technical result, we show how to "amplify" the resilience $f$ at a cost of losing some 
guarantees:

- Input: Two counters with a small $f$; guaranteed to work permanently after stabilization.

- Output: A counter with a large $f$; guaranteed to work only once in a while. This can be then used to jointly run a single instance of consensus and stabilize the output. We show how to obtain such a counter based on simple local consistency checks, timeouts, and threshold voting.

In the end, a recursive application of this scheme allows us to build space-efficient counting algorithms for any $n$ with optimal resilience. At each level of recursion, we need only run a single instance of consensus. As there will be $O(\log f)$ levels of recursion, in total each node participates in only $O(\log f)$ consensus instances.

1.3. Structure. Section 2 reviews prior work on impossibility results and counting algorithms. Section 3 provides a formal description of the basic model of computation and the synchronous counting problem. Section 4 gives the main technical result on resilience boosting, and section 5 applies it to construct fast and communicationefficient algorithms. Section 6 shows how to reduce the number of bits communicated during and after stabilization. Section 7 discusses the pulling model and randomized sampling.

2. Related work. In this section, we first overview impossibility results related to counting, and then discuss both deterministic and randomized algorithms for the counting problem.

Impossibility results. As mentioned, counting is closely related to consensus as reductions exist both ways [12]: consensus can be solved in time $O(T)$ tolerating $f$ faults if and only if counting can be solved in time $O(T)$ tolerating $f$ faults.

With this equivalence in mind, several impossibility results for consensus directly hold for counting as well. First, consensus cannot be solved in the presence of $n / 3$ or more Byzantine failures [27]. Second, any deterministic $f$-resilient consensus algorithm needs to run for at least $f+1$ communication rounds [20]. Third, it is known that the connectivity of the communication network must be at least $2 f+1$ [11]. Finally, any consensus algorithm needs to communicate at least $\Omega(n f)$ bits in total [16].

In terms of communication complexity, no better bound than $\Omega(n f)$ on the total number of communicated bits is known. While nontrivial for consensus, this bound turns out to be trivial for deterministic counting algorithms: a self-stabilizing algorithm needs to verify its output, and to do that, each of the $n$ nodes needs to receive information from at least $f+1=\Omega(f)$ other nodes to be certain that some other nonfaulty node has the same output value. Similarly, no nonconstant lower bounds on the number of state bits nodes are known; however, a nontrivial constant lower bound for the case $f=1$ is known [13].

Prior algorithms. There are several algorithms to the synchronous counting problem, with different trade-offs in terms of resilience, stabilization time, space complexity, communication complexity, and the use of random bits. For a brief summary, see Table 1.

Designing space-efficient randomized algorithms for synchronous counting is fairly straightforward $[13,17,18]$; for example, the nodes can simply choose random states until a clear majority of nodes has the same state, after which they start to follow the majority. Likewise, given a shared coin, one can quickly reach agreement by defaulting to the coin whenever no clear majority is observed [4]. However, existing highly resilient shared coins are very inefficient in terms of communication or need additional assumptions, such as private communication links between correct nodes. 
TABLE 1

Summary of counting algorithms for the case $c=2$. For randomized algorithms, we list the expected stabilization time. ${ }^{(*)}$ The solution from [4] relies on a shared coin-details vary, but all known shared coins with large resilience require large states and messages.

\begin{tabular}{lllll}
\hline Resilience & Stabilization time & State bits & Deterministic & Reference \\
\hline$f<n / 3$ & $O(1)$ & $n^{O(1)}$ & no & {$[4]{ }^{(*)}$} \\
$f<n / 3$ & $O(f)$ & $O(f \log f)$ & yes & {$[14]$} \\
$f<n / 3$ & $2^{2(n-f)}$ & 2 & no & {$[17,18]$} \\
$f<n / 3$ & $\min \left\{2^{2 f+2}+1,2^{O\left(f^{2} / n\right)}\right\}$ & 1 & no & {$[13]$} \\
$f=1, n \geq 4$ & 7 & 2 & yes & {$[13]$} \\
$f=n^{1-o(1)}$ & $O(f)$ & $O\left(\log ^{2} f / \log \log f\right)$ & yes & {$[26]$} \\
\hline$f<n / 3$ & $O(f)$ & $O\left(\log ^{2} f\right)$ & yes & this work \\
\hline
\end{tabular}

Less resilient shared coins are easier to obtain: Resilience $\Theta(\sqrt{n})$ is achieved by each node announcing the outcome of an independent coin flip and locally outputting the (observed) majority value. In addition, $\Omega\left(n / \log ^{2} n\right)$-resilient Boolean functions give fast communication-efficient coins [1]. Designing quickly stabilizing algorithms that are both communication-efficient and space-efficient has turned out to be a challenging task $[13,14,15]$, and it remains open to what extent randomization can help in designing such algorithms.

In the case of deterministic algorithms, algorithm synthesis has been used for computer-aided design of optimal algorithms with resilience $f=1$, but the approach does not scale due to the extremely fast-growing space of possible algorithms [13]. In general, many fast-stabilizing algorithms build on a connection between Byzantine consensus and synchronous counting, but require a large number of states per node [14] due to, e.g., running a large number of consensus instances in parallel. Recently, in one of the preliminary conference reports [26] this paper is based on, we outlined a recursive approach where each node needs to participate in only $O(\log f / \log \log f)$ parallel instances of consensus. However, this approach resulted in suboptimal resilience of $f=n^{1-o(1)}$.

Finally, we note that while counting algorithms are usually designed for the case of a fully connected communication topology, the algorithms can be extended to use in a variety of other graph classes with high enough connectivity [13].

Related problems. Boczkowski, Korman, and Natale [7] studied the synchronous $c$-counting problem (under the name self-stabilizing clock synchronization) with $O(\sqrt{n})$ Byzantine faults in a stochastic communication setting that resembles the pulling model we consider in section 7 . However, their communication model is much more restricted: In every round, each node interacts with at most constantly many nodes which are chosen uniformly at random. Moreover, nodes only exchange messages of size $O(\log c)$ bits.

Without Byzantine (or other types of permanent) faults, self-stabilizing counters and digital clocks have been studied as the self-stabilizing unison problem [2, 8, 21]. However, unlike in the fully connected setting considered in this work, the underlying communication topology in the unison problem is typically assumed to be an arbitrary graph. In our model, in the absence of permanent faults the problem becomes trivial, as nodes may simply reproduce the clock of a predetermined leader. The unison problem has also been studied in asynchronous models $[8,19]$; this variant is also known as self-stabilizing synchronizers [3]. 
3. Preliminaries. In this section, we define the model of computation and the counting problem.

3.1. Model of computation. Here we consider a fully connected synchronous message-passing network. That is, our distributed system consists of a network of $n$ nodes, where each node is a state machine and has communication links to all other nodes in the network. All nodes have a unique identifier from the set $[n]=\{0,1, \ldots, n-1\}$. The computation proceeds in synchronous communication rounds. In each round, all nodes perform the following in a lock-step fashion:

1. broadcast a single message to all nodes,

2. receive messages from all nodes, and

3. update the local state.

We assume that the initial state of each node is arbitrary and there are up to $f$ Byzantine nodes. A Byzantine node may have arbitrary behavior, that is, it can deviate from the protocol in any manner. In particular, the Byzantine nodes can collude together in an adversarial manner and a single Byzantine node can send different messages to different correct nodes.

Algorithms. Formally, we define an algorithm as a tuple $\mathbf{A}=\langle X, g, p\rangle$, where $X$ is the set of all states any node can have, $g:[n] \times X^{n} \rightarrow X$ is the state transition function, and $p:[n] \times X \rightarrow[c]$ is the output function. At each round when node $v$ receives a vector $\mathbf{x}=\left\langle x_{0}, \ldots, x_{n-1}\right\rangle \in X^{n}$ of messages, node $v$ updates it to state $g(v, \mathbf{x})$ and outputs $p\left(v, x_{v}\right)$. As we consider $c$-counting algorithms, the set of output values is the set $[c]=\{0,1, \ldots, c-1\}$ of counter values.

The tuples passed to the state transition function $g$ are ordered according to the node identifiers. In other words, the nodes can identify the sender of a message - this is frequently referred to as source authentication. Moreover, in the basic model, we assume that all nodes simply broadcast their state to all other nodes. Thus, the set of messages is the same as the set of possible states.

Executions. For any set of $\mathcal{F} \subseteq[n]$ of faulty nodes, we define a projection $\pi_{\mathcal{F}}$ that maps any state vector $\mathbf{x} \in X^{n}$ to a configuration $\pi_{F}(\mathbf{x})=\mathbf{e}$, where $e_{v}=*$ if $v \in \mathcal{F}$ and $e_{v}=x_{v}$ otherwise. That is, the values given by Byzantine nodes are ignored and a configuration consists of only the states of correct nodes. A configuration $\mathbf{d}$ is reachable from configuration e if for every correct node $v \notin \mathcal{F}$ there exists some $\mathbf{x} \in X^{n}$ satisfying $\pi_{\mathcal{F}}(\mathbf{x})=\mathbf{e}$ and $g(v, \mathbf{x})=d_{v}$. An execution of an algorithm $\mathbf{A}$ is an infinite sequence of configurations $\xi=\left\langle\mathbf{e}_{0}, \mathbf{e}_{1} \ldots,\right\rangle$ where configuration $\mathbf{e}_{r+1}$ is reachable from configuration $\mathbf{e}_{r}$.

3.2. Synchronous counters and complexity measures. We say that an execution $\xi=\left\langle\mathbf{e}_{0}, \mathbf{e}_{1} \ldots,\right\rangle$ of a counting algorithm $\mathbf{A}$ stabilizes in time $T$ if there is some $k \in[c]$ such that for every correct node $v \in[n] \backslash \mathcal{F}$ it holds that

$$
p\left(v, e_{T+r, v}\right)=r-k \bmod c \text { for all } r \geq 0,
$$

where $e_{T+r, v} \in X$ is the state of node $v$ in round $T+r$.

An algorithm $\mathbf{A}$ is said to be a synchronous c-counter with resilience $f$ that stabilizes in time $T$ if for every $\mathcal{F} \subseteq[n],|\mathcal{F}| \leq f$, all executions of algorithm $\mathbf{A}$ stabilize within $T$ rounds. In this case, we say that the stabilization time $T(\mathbf{A})$ of $\mathbf{A}$ is the minimal such $T$ that all executions of $\mathbf{A}$ stabilize in $T$ rounds. The state complexity of $\mathbf{A}$ is $S(\mathbf{A})=\lceil\log |X|\rceil$, that is, the number of bits required to encode the state of a node between subsequent rounds. For brevity, we will often refer to $\mathcal{A}(n, f, c)$ as the family of synchronous $c$-counters over $n$ nodes with resilience $f$. For 
example, $\mathbf{A} \in \mathcal{A}(4,1,2)$ denotes a synchronous 2-counter (i.e., a binary counter) over four nodes tolerating one failure.

4. Boosting resilience. In this section, we show how to use existing "small" synchronous counters to construct new "large" synchronous counters with a higher resilience $f$ and a larger number of nodes $n$; we call this resilience boosting. We will then apply the idea recursively, with trivial counters as a base case.

4.1. Road map. The high-level idea of resilience boosting is as follows. We start with counters that have a low resilience $f^{\prime}$ and use a small number of nodes $n^{\prime}$. We use such counters to construct a new "weak" counter that has a higher resilience $f>f^{\prime}$ and a large number of nodes $n>n^{\prime}$ but need only behave correctly once in a while for sufficiently long. Once such a weak counter exists, it can be used to provide consistent round numbers for long enough to execute a single instance of a high-resilience consensus protocol. This can be used to reach agreement on the output counter.

Constructing the weak counter. For clarity, we will use here the term strong counter to refer to a self-stabilizing fault-tolerant counter in the usual sense, and the term weak counter to refer to a counter that behaves correctly once in a while. We assume that $f^{\prime}$-resilient strong counters for all $f^{\prime}<f$ already exist, and we show how to construct an $f$-resilient weak counter that behaves correctly for at least $\tau$ rounds. Put slightly more formally, a weak $\tau$-counter satisfies the following property: There exists a round $r$ such that for all correct nodes $v, w \in V \backslash F$ satisfy

- $d(v, r)=d(w, r)$ and

- $d\left(v, r^{\prime}\right)=d\left(v, r^{\prime}-1\right)+1 \bmod \tau$ for all $r^{\prime} \in\{r+1, \ldots, r+\tau-1\}$,

where $d(v, r)$ denotes the value of the weak counter at node $v$ in round $r$. That is, eventually there will be $\tau$ consecutive rounds during which the (weak) counter values agree and are incremented by one modulo $\tau$ every round. However, after these $\tau$ rounds, the counters can behave arbitrarily.

Let $f_{0}+f_{1}+1=f$ and $n_{0}+n_{1}=n$. We take an $f_{0}$-resilient strong $2 \tau$-counter $\mathbf{A}_{0}$ with $n_{0}$ nodes and an $f_{1}$-resilient strong $6 \tau$-counter $\mathbf{A}_{1}$ with $n_{1}$ nodes, and use them to construct an $f$-resilient weak counter with $n$ nodes.

We partition $n$ nodes in disjoint "blocks": block 0 runs $\mathbf{A}_{0}$ with $n_{0}$ nodes and block 1 runs $\mathbf{A}_{1}$ with $n_{1}$ nodes. At least one of the algorithms will eventually stabilize and count correctly. The key challenge is making sure that eventually all correct nodes (in both blocks!) will follow the same correct counter, at least for $\tau$ rounds.

To this end, each block maintains a leader pointer. The leader pointers are changed regularly: block 0 changes its leader pointer every $\tau$ rounds, and block 1 changes its leader pointer every $3 \tau$ rounds. If the leader pointers behave correctly, there will be regular periods of $\tau$ rounds such that both of the leader pointers point to the same correct block.

If we had reliable counters, block $i$ could simply use the current value of counter $\mathbf{A}_{i}$ to determine the current value of its leader pointer. However, one of the counters might misbehave. As a remedy, each node $v$ of block $i$ checks if the output variable of counter $\mathbf{A}_{i}$ increases by 1 in each round. If not, it will consider $\mathbf{A}_{i}$ faulty for $\Theta(\tau)$ rounds. The final output of a node is determined as follows:

- If node $v$ in block $i$ thinks that $\mathbf{A}_{i}$ is faulty, it outputs the current value of counter $\mathbf{A}_{1-i}$.

- Otherwise, it uses the current value of $\mathbf{A}_{i}$ to construct the leader pointer $\ell \in\{0,1\}$, and it outputs the current value of counter $\mathbf{A}_{\ell}$.

Note that the counter $\mathbf{A}_{i}$ might seem to be behaving in a faulty manner if there has 
not been enough time for $\mathbf{A}_{i}$ to stabilize. However, each node $v$ of block $i$ will consider a block to be faulty at most $\Theta(\tau)$ rounds before checking again whether the output of $\mathbf{A}_{i}$ behaves consistently. Thus, if $\mathbf{A}_{i}$ eventually stabilizes, then eventually node $v$ stops considering $\mathbf{A}_{i}$ as faulty for good (at least until the next transient failure).

The above consistency check almost cuts it - except that two nodes $w \neq v$ of block $i$ may have different opinions on the current value of $\mathbf{A}_{i}$. We clear this final hurdle by enlisting the help of all nodes for a majority vote on what the current value of $\mathbf{A}_{i}$ actually is. Essentially, we use threshold voting; this way all nodes that think that $\mathbf{A}_{i}$ behaves correctly will agree on a globally unique counter value $\alpha_{i}$ for $\mathbf{A}_{i}$.

If, for example, block 0 contains at most $f_{0}$ faulty nodes, all of this eventually entails the following:

1. Counter $\mathbf{A}_{0}$ stabilizes, counts correctly, and all correct nodes agree on its counter value $\alpha_{0}$.

2. All correct nodes of block 0 think that block 0 is counting correctly. They use $\alpha_{0}$ to derive the value of the leader pointer. Once in $2 \tau$ rounds, when the $2 \tau$-counter $\alpha_{0}$ wraps around to 0 , the pointer switches to 0 , and the nodes will output the counter value $\alpha_{0}$ for $\tau$ rounds.

3. Some correct nodes of block 1 may think that block 1 is counting correctly for $\Theta(\tau)$ rounds. While this is the case, all of them agree on a value $\alpha_{1}$ that increases by 1 in each round. This value is used to derive the leader pointer of block 1 . Once in $6 \tau$ rounds, when the $6 \tau$-counter $\alpha_{1}$ wraps around to 0 , the pointer will switch to 0 , and the nodes will output the value of $\alpha_{0}$ for $3 \tau$ rounds (as the leader pointer does not change for $3 \tau$ rounds).

4. Some correct nodes of block 1 may detect that block 1 is faulty. Such nodes will output the value of $\alpha_{0}$ for $\Theta(\tau)$ rounds.

5. In summary, eventually there will be $\tau$ consecutive rounds during which all correct nodes output the same counter value $\alpha_{0}$.

The other case (block 1 has at most $f_{1}$ faulty nodes) is analogous.

Using the weak counter. Now we have constructed a counter that will eventually produce a consistent output for at least $\tau$ rounds. We leverage this property to execute the phase king consensus protocol [6] to stabilize the output counters. The protocol will have the following crucial property: if all nodes agree on the output, then even if the round counter becomes inconsistent, the agreement on the output persists. Thus, it suffices for us that $\tau$ is large enough to enable the nodes to consistently execute the phase king algorithm once to reach agreement; $\tau=O(f)$ will do.

The stabilization time on each level is the maximum of the stabilization times of counters $\mathbf{A}_{i}$ plus $O(\tau)=O(f)$; by choosing $f_{1} \approx f_{2} \approx f / 2$, we can thus ensure an overall stabilization time of $O(f)$, irrespectively of the number of recursion levels. Formally, we prove the following theorem.

Theorem 4.1. Let $c, n>1$ and $f<n / 3$. Define $n_{0}=\lfloor n / 2\rfloor, n_{1}=\lceil n / 2\rceil$, $f_{0}=\lfloor(f-1) / 2\rfloor, f_{1}=\lceil(f-1) / 2\rceil$, and $\tau=3(f+2)$. If for $i \in\{0,1\}$ there exist synchronous counters $\mathbf{A}_{i} \in \mathcal{A}\left(n_{i}, f_{i}, c_{i}\right)$ such that $c_{i}=3^{i} \cdot 2 \tau$, then there exists a synchronous c-counter $\mathbf{B} \in \mathcal{A}(n, f, c)$ that

- stabilizes in $T(\mathbf{B})=\max \left\{T\left(\mathbf{A}_{0}\right), T\left(\mathbf{A}_{1}\right)\right\}+O(f)$ rounds, and

- has state complexity of $S(\mathbf{B})=\max \left\{S\left(\mathbf{A}_{0}\right), S\left(\mathbf{A}_{1}\right)\right\}+O(\log f+\log c)$ bits.

We fix the notation of this theorem for the remainder of this section. Moreover, for notational convenience we abbreviate $T=\max \left\{T\left(\mathbf{A}_{0}\right), T\left(\mathbf{A}_{1}\right)\right\}$ and $S=$ $\max \left\{S\left(\mathbf{A}_{0}\right), S\left(\mathbf{A}_{1}\right)\right\}$. 
4.2. Agreeing on a common counter (once in a while). In this part, we construct a counter that will eventually count consistently at all nodes for $\tau$ rounds. The $\tau$-counter then will be used as a common clock for executing the phase king algorithm.

We partition the set of nodes $V=V_{0} \cup V_{1}$ such that $V_{0} \cap V_{1}=\emptyset,\left|V_{0}\right|=n_{0}$ and $\left|V_{1}\right|=n_{1}$. We refer to the set $V_{i}$ as block $i$. For each $i \in\{0,1\}$, the nodes in set $V_{i}$ execute the algorithm $\mathbf{A}_{i}$. In case block $i$ has more than $f_{i}$ faults, we call the block $i$ faulty. Otherwise, we say that block $i$ is correct. By construction, at least one of the blocks is correct. Hence, there is a correct block $i$ for which $\mathbf{A}_{i}$ stabilizes within $T$ rounds, that is, nodes in block $i$ output a consistent $c_{i}$-counter in rounds $r \geq T$.

Lemma 4.2. For some $i \in\{0,1\}$, block $i$ is correct.

Proof. By choice of $f_{i}$, we have $f=f_{0}+f_{1}+1$. Hence, at least one of the sets $V_{i}$ will contain at most $f_{i}$ faults.

Next, we apply the typical threshold voting mechanism employed by most Byzantine tolerant algorithms in order to filter out differing views of counter values that are believed to be consistent. This is achieved by broadcasting candidate counter values and applying a threshold of $n-f$ as a consistency check, which guarantees that at most one candidate value from the set $[c]$ can remain. In case the threshold check fails, a fallback value $\perp \notin[c]$ is used to indicate an inconsistency. This voting scheme is applied for both blocks concurrently, and all nodes participate in the process, so we can be certain that fewer than one third of the voters are faulty.

In addition to passing this voting step, we require that the counters also have behaved consistently over a sufficient number of rounds; this is verified by the obvious mechanism of testing whether the counter increases by 1 each round and counting the number of rounds since the last inconsistency was detected.

In the following, nodes frequently examine a set of values, one broadcast by each node, and determine majority values. Note that Byzantine nodes may send different values to different nodes, that is, it may happen that correct nodes output different values from such a vote. We refer to a strong majority as at least $n-f$ nodes supporting the same value, which is then called the majority value. If a node does not see a strong majority, it outputs the symbol $\perp$ instead. Clearly, this procedure is well-defined for $f<n / 2$.

We will refer to this procedure as a majority vote, and slightly abuse notation by saying "majority vote" when, precisely, we should talk of "the output of the majority vote at node $v$ ". Since we require that $f<n / 3$, the following standard argument shows that for each vote, there is a unique value such that each node either outputs this value or $\perp$.

Lemma 4.3. If $v, w \in V \backslash \mathcal{F}$ both observe a strong majority, they output the same majority value.

Proof. Fix any set $A$ of $n-f$ correct nodes. For $v$ and $w$ to observe strong majorities for different values, for each value $A$ must contain $n-2 f$ nodes supporting it. However, as correct nodes broadcast the same value to each node, this leads to the contradiction that $|A| \geq 2(n-2 f)=n-f+(n-3 f)>n-f=|A|$.

We now put this principle to use. In the following, we will use the notation $x(v, r)$ to refer to the value of local variable $x$ of node $v$ in round $r$. As we consider self-stabilizing algorithms, the nodes themselves are not aware of what is the value of $r$. We introduce the following local variables for each node $v \in V$, block $i \in\{0,1\}$, and round $r>0$ (see Tables 2 and 3 ): 
TABLE 2

The local state variables used in the boosting construction.

\begin{tabular}{lll}
\hline Variable & Range & Description \\
\hline$m_{i}(v, r)$ & {$\left[c_{i}\right]$} & the most frequent value observed for the $\mathbf{A}_{i}$ counter of block $i$ \\
$M_{i}(v, r)$ & {$\left[c_{i}\right] \cup\{\perp\}$} & the result of majority vote on $m_{i}(\cdot, r-1)$ values \\
$w_{i}(v, r)$ & {$\left[c_{1}+1\right]$} & "cooldown counter" that is reset if block $i$ behaved inconsistently \\
\hline$d_{i}(v, r)$ & {$\left[c_{i}\right] \cup\{\perp\}$} & observation on what seems to be the counter output of block $i$ \\
$\ell_{i}(v, r)$ & $\{0,1, \perp\}$ & the value of the "leader pointer" for block $i$ \\
$\ell(v, r)$ & $\{0,1, \perp\}$ & leader pointer used by node $v$ \\
$d(v, r)$ & {$[\tau]$} & once-in-a-while round counter for clocking phase king \\
\hline$a(v, r)$ & {$[c] \cup\{\infty\}$} & the output of the new c-counter we are constructing \\
$b(v, r)$ & $\{0,1\}$ & helper variable for the phase king algorithm \\
\hline
\end{tabular}

TABLE 3

Behavior of local state variables; pointers switch once in $3^{i} \tau$ rounds.

\begin{tabular}{lll}
\hline Variable & Block $i$ is correct & Block $i$ is faulty \\
\hline$m_{i}(v, r)$ & consistent counter & arbitrary values \\
$M_{i}(v, r)$ & consistent counter & $\perp$ or some consistent value \\
$d_{i}(v, r)$ & consistent counter & $\perp$ or some consistent counter \\
$\ell_{i}(v, r)$ & consistent pointer & $\perp$ or some consistent pointer \\
\hline
\end{tabular}

- $m_{i}(v, r)$ stores the most frequent counter value in block $i$ in round $r$, which is determined from the broadcasted output variables of $\mathbf{A}_{i}$ with ties broken arbitrarily,

- $M_{i}(v, r)$ stores the majority vote on $m_{i}(v, r-1)$,

- $w_{i}(v, r)$ is a cooldown counter which is reset to $2 c_{1}$ whenever the node perceives the counter of block $i$ behaving inconsistently, that is, $M_{i}(v, r) \neq M_{i}(v, r-$ $1)+1 \bmod c_{i}$. Note that this test will automatically fail if either value is $\perp$. Otherwise, if the counter behaves consistently, $w_{i}(v, r)=\max \left\{w_{i}(v, r-1)-\right.$ $1,0\}$.

Clearly, these variables can be updated based on the local values from the previous round and the states broadcast at the beginning of the current round. This requires nodes to store $O\left(\log c_{i}\right)=O(\log f)$ bits.

Furthermore, we define the following derived variables for each $v \in V$, block $i \in\{0,1\}$, and round $r$ (see Tables 2 and 3 ):

- $d_{i}(v, r)=M_{i}(v, r)$ if $w_{i}(v, r)=0$, otherwise $d_{i}(v, r)=\perp$,

- $\ell_{i}(v, r)=\left\lfloor d_{i}(v, r) /\left(3^{i} \tau\right)\right\rfloor$ if $d_{i}(v, r) \neq \perp$, otherwise $\ell_{i}(v, r)=\perp$,

- for $v \in V_{i}, \ell(v, r)=\ell_{i}(v, r)$ if $\ell_{i}(v, r) \neq \perp$, otherwise $\ell(v, r)=\ell_{1-i}(v, r)$, and

- $d(v, r)=d_{\ell(v, r)}(v, r) \bmod \tau$ if $\ell(v, r) \neq \perp$, otherwise $d(v, r)=0$.

These can be computed locally, without storing or communicating additional values. The variable $\ell(v, r)$ indicates the block that node $v$ currently considers leader. Note that some nodes may use $\ell_{0}(\cdot, r)$ as the leader pointer while some other nodes may use $\ell_{1}(\cdot, r)$ as the leader pointer, but this is fine:

- all nodes $v$ that use $\ell(v, r)=\ell_{0}(v, r)$ observe the same value $\ell_{0}(\cdot, r) \neq \perp$,

- all nodes $w$ that use $\ell(w, r)=\ell_{1}(w, r)$ observe the same value $\ell_{1}(\cdot, r) \neq \perp$,

- eventually $\ell_{0}(\cdot, r)$ and $\ell_{1}(\cdot, r)$ will point to the same correct block for $\tau$ rounds.

We now verify that $\ell(v, r)$ indeed has the desired properties. To this end, we analyze $d_{i}(v, r)$. We start with a lemma showing that eventually a correct block's counter will be consistently observed by all correct nodes. 
Lemma 4.4. Suppose block $i \in\{0,1\}$ is correct. Then for all $v, w \in V \backslash \mathcal{F}$, and rounds $r \geq R=T+O(f)$ it holds that $d_{i}(v, r)=d_{i}(w, r)$ and $d_{i}(v, r)=d_{i}(v, r-1)+$ $1 \bmod c_{i}$.

Proof. Since block $i$ is correct, algorithm $\mathbf{A}_{i}$ stabilizes within $T\left(\mathbf{A}_{i}\right)$ rounds. As $f_{i}<n_{i} / 3$, we will observe correctly $m_{i}(v, r+1)=m_{i}(v, r)+1 \bmod c_{i}$ for all $r \geq T\left(\mathbf{A}_{i}\right)$. Consequently, $M_{i}(v, r+1)=M_{i}(v, r)+1 \bmod c_{i}$ for all $r \geq T\left(\mathbf{A}_{i}\right)+1$. Therefore, $w_{i}(v, r)$ cannot be reset in rounds $r \geq T\left(\mathbf{A}_{i}\right)+2$, yielding that $w_{i}(v, r)=0$ for all $r \geq T\left(\mathbf{A}_{i}\right)+2+2 c_{1}=T+O(f)$. The claim follows from the definition of variable $d_{i}(v, r)$.

The following lemma states that if a correct node $v$ does not detect an error in a block's counter, then any other correct node $w$ that considers the block's counter correct in any of the last $2 c_{1}$ rounds has a counter value that agrees with $v$.

Lemma 4.5. Suppose for $i \in\{0,1\}, v \in V \backslash \mathcal{F}$, and $r \geq 2 c_{1}=O(f)$ it holds that $d_{i}(v, r) \neq \perp$. Then for each $w \in V \backslash \mathcal{F}$ and each $r^{\prime} \in\left\{r-2 c_{1}+1, \ldots, r\right\}$ either

- $d_{i}\left(w, r^{\prime}\right)=d_{i}(v, r)-\left(r-r^{\prime}\right) \bmod c_{i}$, or

- $d_{i}\left(w, r^{\prime}\right)=\perp$.

Proof. Suppose $d_{i}\left(w, r^{\prime}\right) \neq \perp$. Thus, $d_{i}\left(w, r^{\prime}\right)=M_{i}\left(w, r^{\prime}\right) \neq \perp$. By Lemma 4.3, either $M_{i}\left(v, r^{\prime}\right)=\perp$ or $M_{i}\left(v, r^{\prime}\right)=M_{i}\left(w, r^{\prime}\right)$. However, $M_{i}\left(v, r^{\prime}\right)=\perp$ would imply that $w_{i}\left(v, r^{\prime}\right)=2 c_{1}$ and thus

$$
w_{i}(v, r) \geq w_{i}\left(v, r^{\prime}\right)+r^{\prime}-r=2 c_{1}+r^{\prime}-r>0,
$$

contradicting the assumption that $d_{i}(v, r) \neq \perp$. Thus, $M_{i}\left(v, r^{\prime}\right)=M_{i}\left(w, r^{\prime}\right)=$ $d_{i}\left(w, r^{\prime}\right)$. More generally, we get from $r-r^{\prime}<2 c_{1}$ and $w_{i}(v, r)=0$ that $w_{i}\left(v, r^{\prime \prime}\right) \neq 2 c_{1}$ for all $r^{\prime \prime} \in\left\{r^{\prime}, \ldots, r\right\}$. Therefore, we have that $M_{i}\left(v, r^{\prime \prime}+1\right)=M_{i}\left(v, r^{\prime \prime}\right)+1 \bmod c$ for all $r^{\prime \prime} \in\left\{r^{\prime}, \ldots, r-1\right\}$, implying

$$
d_{i}(v, r)=M_{i}(v, r)=M_{i}\left(v, r^{\prime}\right)+r-r^{\prime}=d_{i}\left(w, r^{\prime}\right)+r-r^{\prime}
$$

proving the claim of the lemma.

The above properties allow us to prove a key lemma: within $T+O(f)$ rounds, there will be $\tau$ consecutive rounds during which the variable $\ell(v, r)$ points to the same correct block for all correct nodes.

LEMmA 4.6. Let $R$ be as in Lemma 4.4. There is a round $r \leq R+O(f)=T+O(f)$ and a correct block $i$ so that for all $v \in V \backslash \mathcal{F}$ and $r^{\prime} \in\{r, \ldots, r+\tau-1\}$ it holds that $\ell\left(v, r^{\prime}\right)=i$.

Proof. By Lemma 4.2, there exists a correct block $i$. Thus by Lemma 4.4, variable $d_{i}(v, r)$ counts correctly during rounds $r \geq R$. If there is no round $r \in\left\{R, \ldots, R+c_{i}-1\right\}$ such that some $v \in V \backslash \mathcal{F}$ has $\ell_{1-i}(v, r) \neq \perp$, then $\ell(v, r)=\ell_{i}(v, r)$ for all such $v$ and $r$ and the claim of the lemma holds true by the definition of $\ell_{i}(v, r)$ and the fact that $d_{i}(v, r)$ counts correctly and consistently.

Hence, assume that $r_{0} \in\left\{R, \ldots, R+c_{i}-1\right\}$ is minimal with the property that there is some $v \in V \backslash \mathcal{F}$ so that $\ell_{1-i}\left(v, r_{0}\right) \neq \perp$. Therefore, $d_{1-i}\left(v, r_{0}\right) \neq \perp$ and, by Lemma 4.5, this implies for all $w \in V \backslash \mathcal{F}$ and all $r \in\left\{r_{0}, \ldots, r_{0}+2 c_{1}-1\right\}$ that either $d_{1-i}(w, r)=\perp$ or $d_{1-i}(w, r)=d_{1-i}\left(v, r_{0}\right)+r-r_{0}$. In other words, there is a "virtual counter" that equals $d_{1-i}\left(v, r_{0}\right)$ in round $r_{0}$ so that during rounds $\left\{r_{0}, \ldots, r_{0}+2 c_{1}-1\right\}$ all $d_{1-i}(\cdot, \cdot)$ variables that are not $\perp$ agree with this counter. 
Consequently, it remains to show that both $\ell_{i}$ and the variable $\ell_{1-i}$ derived from this virtual counter are equal to $i$ for $\tau$ consecutive rounds during the interval $I=\left\{r_{0}, \ldots, r_{0}+2 c_{1}-1\right\}$, as then $\ell\left(v, r^{\prime}\right)=i$ for $v \in V \backslash \mathcal{F}$ and all such rounds $r^{\prime}$.

Clearly, the $c_{1}$-counter consecutively counts from 0 to $c_{1}-1$ at least once during the interval $I=\left\{r_{0}, \ldots, r_{0}+2 c_{1}-1\right\}$. Recalling that $c_{1}=6 \tau$, we see that $\ell_{1}(v, r)=i$ for all $v \in V \backslash \mathcal{F}$ with $\ell_{1}(v, r) \neq \perp$ for some interval $I_{1} \subset I$ of $3 \tau$ consecutive rounds. As $c_{0}=2 \tau$, we have that $\ell_{0}(v, r)=i$ for all $v \in V \backslash \mathcal{F}$ with $\ell_{0}(v, r) \neq \perp$ for $\tau$ consecutive rounds during this subinterval $I_{1}$. Thus, we have an interval $I_{0}=\{r, \ldots, r+\tau-1\} \subseteq I_{1}$ such that for all $r^{\prime} \in I_{0}$ we have $\ell_{0}\left(v, r^{\prime}\right), \ell_{1}\left(v, r^{\prime}\right) \in\{i, \perp\}$ and $\ell_{0}\left(v, r^{\prime}\right) \neq \perp$ or $\ell_{1}\left(v, r^{\prime}\right) \neq \perp$ yielding $\ell\left(v, r^{\prime}\right)=i$ for each correct node. Because $r<r_{0}+2 c_{1}-1<R+3 c_{1}=T+O(f)$, this completes the proof.

Using the above lemma, we get a counter where all nodes eventually count correctly and consistently modulo $\tau$ for at least $\tau$ rounds.

Corollary 4.7. There is a round $r=T+O(f)$ so that for all $v, w \in V \backslash \mathcal{F}$ it holds that

1. $d(v, r)=d(w, r)$ and

2. for all $r^{\prime} \in\{r+1, \ldots, r+\tau-1\}$ we have $d\left(v, r^{\prime}\right)=d\left(v, r^{\prime}-1\right)+1 \bmod \tau$.

Proof. By Lemma 4.6, there is a round $r=T+O(f)$ and a correct block $i$ such that for all $v \in V \backslash \mathcal{F}$ we have $\ell\left(v, r^{\prime}\right)=i$ for all $r^{\prime} \in\{r, \ldots, r+\tau-1\}$. Moreover, $r$ is sufficiently large to apply Lemma 4.4 to $d_{i}\left(v, r^{\prime}\right)=d\left(v, r^{\prime}\right)$ for $r^{\prime} \in\{r+1, \ldots, r+\tau-1\}$, yielding the claim.

4.3. Reaching consensus. Corollary 4.7 guarantees that all correct nodes eventually agree on a common counter for $\tau$ rounds, i.e., we have a weak counter. We will now use the weak counter to construct a strong counter.

Our construction uses a non-self-stabilizing consensus algorithm. The basic idea is that the weak counter serves as the "round counter" for the consensus algorithm. Hence we will reach agreement as soon as the weak counter is counting correctly. The key challenge is to make sure that agreement persists even if the counter starts to misbehave. It turns out that a straightforward adaptation of the classic phase king protocol [6] does the job. The algorithm has the following properties:

- the algorithm tolerates $f<n / 3$ Byzantine failures,

- the running time of the algorithm is $O(f)$ rounds and it uses $O(\log c)$ bits of state,

- if node $k$ is correct, then agreement is reached if all correct nodes execute rounds $3 k, 3 k+1$, and $3 k+2$ consecutively in this order,

- once agreement is reached, it will persist even if nodes execute different rounds in arbitrary order.

We now describe the modified phase king algorithm that will yield a $c$-counting algorithm. Denote by $a(v, r) \in[c] \cup\{\infty\}$ the output value of the algorithm at round $r$. Here $\infty$ is used as a "reset state" similarly to $\perp$ in the previous section. There is also an auxiliary binary value $b(v, r) \in\{0,1\}$. Define the following short-hand for the increment operation modulo $c$ :

$$
x \oplus 1= \begin{cases}x+1 \bmod c & \text { if } x \neq \infty \\ \infty & \text { if } x=\infty .\end{cases}
$$

For $k \in[f+2]$, we define the instruction sets listed in Table 4 . Recall that in the model of computation that we use in this work, in each round all nodes first 
TABLE 4

The instruction sets for node $v \in V$ in the phase king protocol.

\begin{tabular}{|c|c|}
\hline Set & Instructions for round $r>0$ \\
\hline$I_{3 k}:$ & $\begin{array}{l}\text { 0a. If fewer than } n-f \text { nodes sent } a(v, r-1) \text {, set } a(v, r)=\infty \text {. } \\
\text { 0b. Otherwise, } a(v, r)=a(v, r-1) \oplus 1\end{array}$ \\
\hline$I_{3 k+1}:$ & $\begin{array}{l}\text { 1a. Let } z_{j}=|\{u \in V: a(u, r-1)=j\}| \text { be the number of } j \text { values received. } \\
\text { 1b. If } z_{a(v, r-1)} \geq n-f \text {, set } b(v, r)=1 \text {. Otherwise, set } b(v, r)=0 \text {. } \\
\text { 1c. Let } z=\min \left\{j: z_{j}>f\right\} \text {. } \\
\text { 1d. Set } a(v, r)=z \oplus 1\end{array}$ \\
\hline$I_{3 k+2}:$ & $\begin{array}{l}\text { 2a. If } a(v, r-1)=\infty \text { or } b(v, r-1)=0 \text {, set } a(v, r)=\min \{c-1, a(k, r-1)\} \oplus 1 . \\
\text { 2b. Otherwise, } a(v, r)=a(v, r-1) \oplus 1 . \\
\text { 2c. Set } b(v, r)=1 .\end{array}$ \\
\hline
\end{tabular}

broadcast their current state (in particular, the current value of $a$ ), then they receive the messages, and finally they update their local state. The instruction sets pertain to the final part- how to update the local state variables $a$ and $b$ based on the messages received from the other nodes.

First, we show that if the instruction sets are executed in the right order by all correct nodes for a correct leader node $k \in[f+2]$, then agreement on a counter value is established.

Lemma 4.8. Suppose that for some correct node $k \in[f+2]$ and a round $r>2$, all nonfaulty nodes execute instruction sets $I_{3 k}, I_{3 k+1}$, and $I_{3 k+2}$ in rounds $r-2, r-1$, and $r$, respectively. Then $a(v, r)=a(u, r) \neq \infty$ for any two correct nodes $u, v \in V$. Moreover, $b(v, r+1)=1$ at each correct node $v \in V$.

Proof. This is essentially the correctness proof for the phase king algorithm. Without loss of generality, we can assume that the number of faulty nodes is exactly $f$. Since we have $f<n / 3$, it is not possible that two correct nodes $u, v \in V \backslash \mathcal{F}$ both satisfy $a(v, r-2) \neq a(u, r-2)$ and $a(v, r-2), a(u, r-2) \in[c]$ : otherwise, on round $r-2$, nodes $u$ and $v$ would have observed different majority values contradicting Lemma 4.3. Therefore, there exists some $x \in[c]$ such that $a(v, r-2) \in\{x, \infty\}$ for all $v \in V \backslash \mathcal{F}$. Checking $I_{3 k+1}$ we get that $a(v, r-1) \in\{x+1 \bmod c, \infty\}$, as no node can see values other than $x$ or $\infty$ more than $f$ times when executing instruction $1 \mathrm{c}$.

To prove the claim, it remains to consider two cases when executing instructions in $I_{3 k+2}$. In the first case, all nonfaulty nodes execute instruction $2 \mathrm{a}$ on round $r$. Then $a(u, r)=a(v, r)=\min \{c-1, a(k, r-1)\} \oplus 1 \in[c]$ for any $u, v \in V \backslash \mathcal{F}$.

In the second case, there is some node $v$ not executing instruction 2a. Hence, $a(v, r-1) \neq \infty$ and $b(v, r-1)=1$, implying that $v$ computed $z_{a(v, r-2)} \geq n-f$ on round $r-1$. Consequently, at least $n-2 f>f$ correct nodes $u$ satisfy $a(u, r-2)=$ $a(v, r-2) \neq \infty$. We can now infer that $a(u, r-1)=a(v, r-1)=a(v, r-2)+1 \bmod c$ for all correct nodes $u$ : instruction $1 \mathrm{c}$ must evaluate to $a(v, r-1) \in[c]$ at all correct nodes, because we know that no correct node $u$ satisfies that both $a(u, r-2) \neq a(v, r-2)$ and $a(u, r-2) \neq \infty$. This implies that $a(u, r)=a(v, r) \neq \infty$ for all correct nodes $u$, regardless of whether they execute instruction 2a. Trivially, $b(v, r)=1$ at each correct node $v$ due to instruction $2 \mathrm{c}$.

Next, we argue that once agreement is established, it persists - it does not matter any more which instruction sets are executed.

Lemma 4.9. Assume that $a(v, r)=x \in[c]$ and $b(v, r)=1$ for all correct nodes $v$ in some round $r$. Then $a(v, r+1)=x+1 \bmod c$ and $b(v, r+1)=1$ for all correct 
nodes $v$.

Proof. Each node observes at least $n-f$ nodes with counter value $x \in[c]$, and hence at most $f$ nodes with some value $y \neq x$. Let $v$ be a correct node and consider all possible instruction sets it may execute.

First, consider the case where instruction set $I_{3 k}$ is executed. In this case, $v$ increments $x$, resulting in $a(v, r+1)=x+1 \bmod c$ and $b(v, r+1)=1$. Second, executing $I_{3 k+1}$, node $v$ evaluates $z_{x} \geq n-f$ and $z_{y} \leq f$ for all $y \neq x$. Hence it sets $b(v, r+1)=1$ and $a(v, r+1)=x+1 \bmod c$. Finally, when executing $I_{3 k+2}$, node $v$ skips instruction $2 \mathrm{a}$ and sets $a(v, r+1)=x+1 \bmod c$ and $b(v, r+1)=1$.

4.4. Proof of Theorem 4.1. We now have all the building blocks to devise an $f$-resilient $c$-counter running on $n$ nodes. The idea is as follows: First, we use the construction given in subsection 4.2 to get a weak $\tau$-counter that eventually counts correctly for $\tau=3(f+2)$ rounds. Concurrently, all nodes execute the modified phase king algorithm given in subsection 4.3 which by Lemmas 4.8 and 4.9 guarantees that all nodes will establish and maintain agreement on the output variable for the $c$-counter.

Theorem 4.1. Let $c, n>1$ and $f<n / 3$. Define $n_{0}=\lfloor n / 2\rfloor, n_{1}=\lceil n / 2\rceil$, $f_{0}=\lfloor(f-1) / 2\rfloor, f_{1}=\lceil(f-1) / 2\rceil$, and $\tau=3(f+2)$. If for $i \in\{0,1\}$ there exist synchronous counters $\mathbf{A}_{i} \in \mathcal{A}\left(n_{i}, f_{i}, c_{i}\right)$ such that $c_{i}=3^{i} \cdot 2 \tau$, then there exists a synchronous c-counter $\mathbf{B} \in \mathcal{A}(n, f, c)$ that

- stabilizes in $T(\mathbf{B})=\max \left\{T\left(\mathbf{A}_{0}\right), T\left(\mathbf{A}_{1}\right)\right\}+O(f)$ rounds, and

- has state complexity of $S(\mathbf{B})=\max \left\{S\left(\mathbf{A}_{0}\right), S\left(\mathbf{A}_{1}\right)\right\}+O(\log f+\log c)$ bits.

Proof. First, we apply the construction underlying Corollary 4.7. Then we have every node $v \in V$ in each round $r$ execute the instructions for round $d(v, r)$ of the phase king algorithm from subsection 4.3. It remains to show that this yields a correct algorithm $\mathbf{B}$ with stabilization time $T(\mathbf{B})=T+O(f)$ and state complexity $S(\mathbf{B})=S+O(\log f+\log c)$, where $T=\max \left\{T\left(\mathbf{A}_{i}\right)\right\}$ and $S=\max \left\{S\left(\mathbf{A}_{i}\right)\right\}$.

By Corollary 4.7, there exists a round $r=T+O(f)$ so that the variables $d(v, r)$ behave as a consistent $\tau$-counter during rounds $\{r, \ldots, r+\tau-1\}$ for all $v \in V \backslash \mathcal{F}$. As there are at most $f$ faulty nodes, there exist at least two correct nodes $v \in[f+2]$. Since $\tau=3(f+2)$, then for at least one correct node $k \in[f+2] \backslash \mathcal{F}$, there is a round $r \leq r_{k} \leq r+\tau-3$ such that $d\left(w, r_{k}+h\right)=3 k+h$ for all $w \in V \backslash \mathcal{F}$ and $h \in\{0,1,2\}$. Therefore, by Lemmas 4.8 and 4.9, the output variables satisfy $a\left(v, r^{\prime}\right)=a\left(w, r^{\prime}\right) \in[c]$ for all correct nodes and rounds $r^{\prime} \geq r_{k}+3$. Thus, the algorithm stabilizes in $r_{v}+3 \leq r+\tau=r+O(f)=T+O(f)$ rounds.

The bound for the state complexity follows from the facts that, at each node, we need at most $S$ bits to store the state of $\mathbf{A}_{i}$ and $O(\log \tau+\log c)=O(\log f+\log c)$ bits to store the variables listed in Table 2 .

5. Deterministic counting. In this section, we use the construction given in the previous section to obtain algorithms that need only a small number of state bits. Essentially, all that remains is to recursively apply Theorem 4.1. Each step of the recursion roughly doubles the resilience in an optimal manner: If we start with an optimally resilient algorithm, we get a new algorithm with higher, but still optimal, resilience. Therefore, to get any desired resilience of $f>0$, it suffices to repeat the recursion for $\Theta(\log f)$ many steps. Figure 1 illustrates how we can recursively apply Theorem 4.1.

We now analyze the correctness, time, and state complexity of the resulting algorithms. 


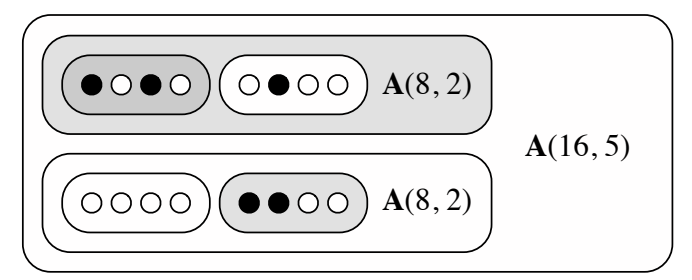

FIG. 1. An example of how to recursively construct a 5-resilient algorithm running on 16 nodes. The small circles represent the nodes. Each group of four nodes runs a 1-resilient counter $\mathbf{A}(4,1)$. On top of this, each larger group of eight nodes runs a 2-resilient counter $\mathbf{A}(8,2)$ attained from the first step of recursion. At the topmost layer, all of the 16 nodes run a 5-resilient counter $\mathbf{A}(16,5)$. Faulty nodes are black and faulty blocks are gray.

THEOREM 1.1. For any integers $c, n>1$ and $f<n / 3$, there exists a deterministic $f$-resilient synchronous c-counter that runs on $n$ nodes, stabilizes in $O(f)$ rounds, and uses $O\left(\log ^{2} f+\log c\right)$ bits to encode the state of a node.

Proof. We show the claim by induction on $f$. The induction hypothesis is that for all $f>f^{\prime} \geq 0, c>1$, and $n^{\prime}>3 f^{\prime}$, we can construct $\mathbf{B} \in \mathcal{A}\left(f^{\prime}, n^{\prime}, c\right)$ with

$$
T(\mathbf{B}) \leq 1+\alpha f^{\prime} \sum_{k=0}^{\left\lceil\log f^{\prime}\right\rceil}(1 / 2)^{k} \quad \text { and } \quad S(\mathbf{B}) \leq \beta\left(\log ^{2} f^{\prime}+\log c\right)
$$

where $\alpha$ and $\beta$ are sufficiently large constants and for $f^{\prime}=0$ the sum is empty, that is, $T(\mathbf{B}) \leq 1$. As $\sum_{k=0}^{\infty}(1 / 2)^{k}=2$, the time bound will be $O\left(f^{\prime}\right)$.

Note that for $f \geq 0$ it is sufficient to show the claim for $n(f)=3 f+1$, as we can easily generalize to any $n>n(f)$ by running $\mathbf{B}$ on the first $n(f)$ nodes and letting the remaining nodes follow the majority counter value among the first $n(f)$ nodes executing the algorithm; this increases the stabilization time by one round and induces no memory overhead.

For the base case, observe that a 0 -resilient $c$-counter of $n(0)=1$ node is trivially given by the node having a local counter. It stabilizes in 0 rounds and requires $\lceil\log c\rceil$ state bits. As pointed out above, this implies a 0-resilient $c$-counter for any $n$ with stabilization time 1 and $\lceil\log c\rceil$ bits of state.

For the inductive step to $f$, we apply Theorem 4.1 with the parameters $n_{0}=\lfloor n / 2\rfloor$, $\left.\left.n_{1}=\lceil n / 2\rceil, f_{0}=\lfloor(f-1) / 2)\right\rfloor, f_{1}=\lceil(f-1) / 2)\right\rceil, \tau=3(f+2)$, and $c_{i}=3^{i} \cdot 2 \tau$. Since $f_{i} \leq f / 2$ and $n_{i}>3 f_{i}$, for $i \in\{0,1\}$, the induction hypothesis gives us algorithms $\mathbf{A}_{i}\left(n_{i}, f_{i}, c_{i}\right)$. Now by applying Theorem 4.1 we get an algorithm $\mathbf{B}$ with

$$
\begin{aligned}
T(\mathbf{B}) & =\max \left\{T\left(\mathbf{A}_{0}\right), T\left(\mathbf{A}_{1}\right)\right\}+O(f) \\
& \leq 1+\frac{\alpha f}{2} \sum_{k=0}^{\lceil\log f / 2\rceil}\left(\frac{1}{2}\right)^{k}+O(f) \\
& =1+\alpha f \sum_{k=1}^{\lceil\log f\rceil}\left(\frac{1}{2}\right)^{k}+O(f) \\
& \leq 1+\alpha f \sum_{k=0}^{\lceil\log f\rceil}\left(\frac{1}{2}\right)^{k},
\end{aligned}
$$


where in the second to last step we use that $\alpha$ is a sufficiently large constant. Since the sum is at most 2, we get that $T(\mathbf{B})=O(f)$. Moreover, the state complexity is bounded by

$$
\begin{aligned}
S(\mathbf{B}) & =\max \left\{S\left(\mathbf{A}_{0}\right), S\left(\mathbf{A}_{1}\right)\right\}+O(\log f+\log c) \\
& \leq \beta\left(\log ^{2} \frac{f}{2}+\log \frac{f}{2}\right)+O(\log f+\log c) \\
& \leq \beta\left(\log ^{2} f+\log c\right),
\end{aligned}
$$

where we exploit that $\beta$ is a sufficiently large constant. Hence, $S(\mathbf{B})=O\left(\log ^{2} f+\log c\right)$, the induction step succeeds, and the proof is complete.

6. Reducing the number of bits communicated. In this section, we discuss how to reduce the number of bits broadcast by a node after stabilization. We consider the following extension of the model of computation: Instead of a node always broadcasting its current state, we allow it to broadcast an arbitrary message (including an empty message) each round. Formally, this entails that we extend the definition of an algorithm by (1) introducing a new function $\mu:[n] \times X \rightarrow \mathcal{M}$ that maps the current state $x$ to a message $\mu(x)$ which is broadcast and (2) modify the state transition function to map the old internal state and the vector of received messages to a new state, that is, the new state transition function has the form $g^{\prime}:[n] \times X \times \mathcal{M}^{n} \rightarrow X$.

First, we show how to construct counters that only send $O(1+B \log B)$ bits every $\kappa$ rounds, where $B=O(\log c / \log \kappa)$, while increasing the stabilization time only by an additive $O(\kappa)$ term, where $\kappa=\Omega(f)$ is a parameter. In particular, we show that for polynomial-sized counters with optimal resilience, the algorithm need only communicate an asymptotically optimal number of bits after stabilization.

Corollary 6.1. For any $n>1$ and $c=n^{O(1)}$ that is an integer multiple of $n$, there exists a synchronous c-counter that runs on $n$ nodes, has optimal resilience $f=\lfloor(n-1) / 3\rfloor$, stabilizes in $\Theta(n)$ rounds, requires $O\left(\log ^{2} n\right)$ bits to encode the state of a node, and for which after stabilization correct nodes broadcast aysmptotically optimal $O(1)$ bits per $\Theta(n)$ rounds.

We start by outlining the high-level idea of the approach, then give a detailed description of the construction we use, and finally prove the main results of this section.

6.1. High-level idea. The techniques we use are very similar to the ones we used for deriving Theorem 1.1. Essentially, we devise a "silencing wrapper" for algorithms given by Theorem 1.1. Let $\mathbf{A}$ be such a counting algorithm. The high-level idea and the key ingredients are the following:

- The goal is that nodes eventually become happy: they assume stabilization has occurred and check for counter consistency only every $\kappa$ rounds (as self-stabilizing algorithms always need to verify their output).

- Happy nodes do not execute the underlying algorithm A.

- Using a cooldown counter with similar effects as shown in Lemma 4.5, we enforce that all happy nodes output consistent counters.

- We override the phase king instruction of $\mathbf{A}$ if at least $n-2 f \geq f+1$ nodes claim to be happy and propose a counter value $x$. In that case nodes adjust their counter output to match $x$. If there is no strong majority of happy nodes supporting a counter value, either all nodes become unhappy or all correct nodes reach agreement and start counting correctly. 
- If all correct nodes are unhappy, they execute A "as is" reaching agreement eventually.

- The counters are used to make all nodes concurrently switch their state to being happy, in a way that does not interfere with the above stabilization process.

We will show that happy nodes can communicate their counter values very efficiently in a manner that self-stabilizes within $\kappa$ rounds. As their counter increases by 1 modulo $c$ every round (or they become unhappy), they can use multiple rounds to encode a counter value; the recipient simply counts locally in the meantime.

6.2. The silencing wrapper. Let $\mathbf{A} \in \mathcal{A}(n, f, c)$ be an algorithm given by Theorem 1.1, and let $c=j \kappa$ for any $j>0$ and $\kappa>T(\mathbf{A})$. We use the short-hand $T=T(\mathbf{A})$ throughout this section. Let $a(v, r)$ be the output of the synchronous counting algorithm for node $v$ in round $r$. Recall that by a strong majority we mean that at least $n-f$ received messages support a value. We now modify $\mathbf{A}$ so that it meets the additional requirement of little communication after stabilization.

We introduce two new variables: a cooldown counter $t(v, r) \in[T+1]$ and a "happiness" indicator $h(v, r) \in\{0,1\}$. These are updated according to the following rules in every round $r>0$ :

1. Set $t(v, r)=T$ if there was no strong majority of nodes $w$ with $a(w, r-1)=$ $a(v, r-1)$ or $a(v, r) \neq a(v, r-1)+1 \bmod c$. Otherwise, decrement the counter, that is, $t(v, r)=\max \{0, t(v, r-1)-1\}$.

2. Set $h(v, r)=0$ if $h(v, r-1)=1$, but there was no strong majority of nodes $w$ with $h(w, r-1)=1$ and $a(w, r-1)=a(v, r-1)$, or if $t(v, r)>0$. Set $h(v, r)=1$ if $t(v, r-1)=0$ and $a(v, r-1)=0 \bmod \kappa$. Otherwise, $h(v, r)=h(v, r-1)$.

3. If $h(v, r)=0$, execute a single step of $\mathbf{A}$ except for the phase king instructions given in Table 4 . The counter value $a(v, r+1)$ is updated according to the next rule.

4. If one receives $n-2 f$ times a value $a(w, r)=x$ from nodes with $h(w, r)=1$, set $a(v, r+1)=x+1 \bmod c$; if there are two such values $x$, it does not matter which is chosen. Otherwise, execute only the phase king instructions of $\mathbf{A}$ given in Table 4 as indicated by the once-in-a-while round counter $d(v, r)$ as usual; in particular, this determines $a(v, r+1)$.

In the following, we say that a node $v \in V \backslash \mathcal{F}$ with value $h(v, r)=1$ is happy in round $r$ and unhappy if $h(v, r)=0$. Moreover, the counters converge in round $r$ if for all $v, w \in V \backslash \mathcal{F}$, it holds that $a(v, r)=a(w, r)$. The idea is to show that not only do the counters converge (and then count correctly), but also all correct nodes become happy. As a happy node that remains happy simply increases its counter value by 1 modulo $c$, there is no need to explicitly communicate this except for verification purposes. It is straightforward to exploit this to ensure that the algorithm communicates very little (explicitly) once all nodes are happy; we will discuss this after showing stabilization of the routine.

6.3. Proof of stabilization. Let us first establish that if the counters converge, they will keep counting correctly and correct nodes will become happy within $O(\kappa+T)$ additional rounds for any parameter $\kappa>T$.

LEMma 6.2. If the counters converge in round $r$, then $a\left(u, r^{\prime}\right)=a\left(v, r^{\prime}\right)=a(u, r)+$ $\left(r-r^{\prime}\right) \bmod c$ for all $u, v \in V \backslash \mathcal{F}$ and $r^{\prime} \geq r$.

Proof. Since the counters have converged, there is a strong majority of nodes supporting the same value. Hence, variable $a\left(u, r^{\prime}\right)$ is updated according to rule 4 . 
As all counter values from correct nodes are identical, it does not matter whether these nodes are happy or not; either way, the counters are increased by 1 modulo $c$ (cf. Lemma 4.9).

LEMma 6.3. If the counters converge in round $r$, then for all rounds $r^{\prime} \geq r+T+\kappa$ and all nodes $v \in V \backslash \mathcal{F}$ we have $h\left(v, r^{\prime}\right)=1$.

Proof. By Lemma 6.2, the agreement on output values will persist once reached. Hence, at all nodes $v \in V \backslash \mathcal{F}$ we have $t\left(v, r^{\prime}\right)=0$ in all rounds $r^{\prime} \geq r+T$ by rule 1 . Therefore, there is a round $r^{\prime} \leq r+T+\kappa$ so that $t\left(v, r^{\prime}\right)=0$ and $a\left(v, r^{\prime}\right)=0 \bmod \kappa$ at all such $v$. Consequently, all correct nodes jointly set $h\left(v, r^{\prime}+1\right)=1$. By induction on the round number, we see that no such node sets $h\left(v, r^{\prime \prime}\right)=0$ for $r^{\prime \prime}>r^{\prime}+1$, as there is always a strong majority of $n-f$ happy and correct nodes supporting the (joint) counter value.

We now proceed to show that the counters converge within $O(\kappa+T)$ rounds. The first step is to observe that if no correct node is happy, then algorithm $\mathbf{A}$ is run without modification, and hence, the counters converge in $T$ rounds.

Lemma 6.4. Let $r \geq T$. If for all $v \in V \backslash \mathcal{F}$ and $r^{\prime} \in\{r-T+1, \ldots, r\}$, we have $h\left(v, r^{\prime}\right)=0$, then the counters converge in round $r+1$.

Proof. Since $h\left(v, r^{\prime}\right)=0$, each node $v$ applies rule 3 in any such round $r^{\prime}$. As there are no happy nodes in round $r^{\prime}$, a node can never receive the same counter value from more than $f$ nodes that (claim to be) happy. Hence, rule 4 boils down to just updating $a\left(v, r^{\prime}\right)$ according to the rules of $\mathbf{A}$. As $T=T(\mathbf{A})$, algorithm $\mathbf{A}$ stabilizes and thus $a(v, r)=a(w, r)$ for all $v, w \in V \backslash \mathcal{F}$.

To deal with the case that some nodes may be happy (which entails that not all nodes may execute $\mathbf{A}$ correctly, destroying its guarantees), we argue that ongoing happiness also implies that the counters converge. To this end, we first show that the cooldown counters $t(v, r)$ ensure that correct nodes whose counters are 0 count correctly and agree on their counter values. This is shown analogously to Lemma 4.5.

Lemma 6.5. Let $r>T$ and $v, w \in V \backslash \mathcal{F}$. If $t(v, r)=t\left(w, r^{\prime}\right)=0$ for $r^{\prime} \in$ $\{r-T+1, \ldots, r\}$, then $a(v, r)=a\left(w, r^{\prime}\right)+r-r^{\prime} \bmod c$.

Proof. Since $t(v, r)=0$, by rule 1 it holds that $t\left(v, r^{\prime}\right) \leq r-r^{\prime}<T$. Hence, both $v$ and $w$ saw a strong majority of nodes $u$ with $a\left(u, r^{\prime}-1\right)=a\left(v, r^{\prime}-1\right)$ and $a\left(u, r^{\prime}-1\right)=a\left(w, r^{\prime}-1\right)$, respectively. By Lemma 4.3, it follows that $a\left(v, r^{\prime}-\right.$ $1)=a\left(w, r^{\prime}-1\right)$. Likewise, $t\left(v, r^{\prime \prime}\right) \neq T$ for rounds $r^{\prime}<r^{\prime \prime} \leq r$, implying that $a(v, r)=a\left(v, r^{\prime}\right)+r-r^{\prime} \bmod c$, and $a\left(w, r^{\prime}\right)=a\left(w, r^{\prime}-1\right)+1 \bmod c=a\left(v, r^{\prime}\right)$.

Except for the initial rounds, the above lemma implies that happy nodes always have the same counter value: By rule 2, a node $v$ with $h(v, r)=1$ must have $t(v, r)=0$. A node remaining happy thus entails that every node receives the same counter value from at least $n-2 f \geq f+1$ happy nodes, and no other counter value with the same property may be perceived. In other words, a node staying happy implies that the counters converge.

Lemma 6.6. If $h(v, r-1)=h(v, r)=1$ for some $v \in V \backslash \mathcal{F}$ and $r>3$, then the counters converge in round $r+1$.

Proof. By rule 2, any node $w$ with $h(v, r)=1$ satisfies $t(w, r)=0$. We apply Lemma 6.5 to see that, for any $w \in V \backslash \mathcal{F}$ that is happy in round $r-1$, we have that $a(v, r-1)=a(w, r-1)$. As $h(v, r)=h(v, r-1)=1$, node $v$ observed a strong majority of happy nodes $w$ with $a(v, r-1)=a(w, r-1)$ in round $r-1$, implying 
that all nodes received this counter value from at least $n-2 f \geq f+1$ happy nodes. Together with rule 4, these observations imply that $a(u, r)=a(v, r-1)+1 \bmod c$ for all $u \in V \backslash \mathcal{F}$.

Using these lemmas and the fact that nodes may become happy only after counting consistently for sufficiently long and when their counters are 0 modulo $\kappa>T$, we can show that the counters converge in all cases.

LEMma 6.7. Within $O(\kappa)$ rounds, the counters converge.

Proof. Either all $v \in V \backslash \mathcal{F}$ with $h(v, 3)=1$ set $h(v, 4)=0$ or Lemma 6.6 shows the claim. If there are no nodes $v$ with $h(v, r)=1$ for $r \in\{4, \ldots, T+3\}$, then Lemma 6.4 shows the claim. Hence, assume that there is some node $v$ with $h(v, r)=1 \neq h(v, r-1)$ for some minimal $r \in\{4, \ldots, T+3\}$. Again, either $h(v, r+1)=0$ for all such nodes or we can apply Lemma 6.6; thus assume the former in the following.

Suppose for contradiction that there is a node $w$ with $h\left(w, r^{\prime}\right)=1$ for a minimal $r^{\prime} \in\{r+1, \ldots, r+T\}$. As $r^{\prime}$ is minimal and all nodes with $h(v, r)=1$ have $h(v, r+1)=0$, it must hold that $h\left(w, r^{\prime}-1\right)=0$. Hence, $t\left(w, r^{\prime}-1\right)=0=t(v, r-1)$. By Lemma 6.5, this implies that $a\left(w, r^{\prime}-1\right)=a(v, r-1)+r-r^{\prime} \bmod c$. However, $\kappa>T, 0<r-r^{\prime} \leq T$, and $a(v, r-1)=0 \bmod \kappa$, implying that $a\left(w, r^{\prime}-1\right) \neq 0 \bmod \kappa$, which (by rule 2) is a contradiction to $h\left(w, r^{\prime}\right)=1 \neq h\left(w, r^{\prime}-1\right)$.

We conclude that $h\left(v, r^{\prime}\right)=0$ for all $v$ and $r^{\prime} \in\{r+1, \ldots, r+T\}$. The claim follows by applying Lemma 6.4.

We now can conclude that within $O(\kappa)$ rounds, the algorithm stabilizes in the sense that all nodes become happy and count correctly and consistently.

COROLlary 6.8. There exists a round $R=O(\kappa)$ such that for all $v \in V \backslash \mathcal{F}$ and $r \geq R$, it holds that $h(v, r)=1$, and $a(v, r)=a(v, r-1)+1 \bmod c$, and $a(v, r)=a(w, r)$ for all $w \in V \backslash \mathcal{F}$.

Proof. By Lemma 6.7 we get that there exists a round $r^{\prime}=O(\kappa)$ in which the counters converge. Since $r^{\prime}+T+\kappa=O(\kappa)$, happiness follows from Lemma 6.3 and agreement follows from Lemma 6.2.

6.4. Reducing the communication complexity after stabilization. As noted earlier, the counter variables for happy nodes count modulo $c$. Hence, it is trivial to deduce the counter value of a happy node from its counter value in an earlier round. Moreover, happy nodes do not execute algorithm A. Therefore, we can change the encoding of the happy nodes' counter values to reduce the communication complexity after stabilization.

Corollary 6.9. Suppose happy nodes communicate their counter values by any method that stabilizes in $\kappa$ rounds, then the algorithm presented in this section retains its properties, except that its stabilization time increases by an additive $\kappa$ rounds.

The above immediately implies that happy nodes $v$ could simply transmit the $a(v, r)$ only in rounds $r$ when $a(v, r) \bmod \kappa=0$ and perform no other communication. The fact that $v$ does not transmit readily implies that it is happy, permitting to derive its counter value by counting from the most recent value $v$ transmitted. Moreover, by Lemma 6.5 the output counters of happy nodes agree after $O(1)$ rounds. Thus, a single local counter suffices for verification yielding a cost of using only $\lceil\log c\rceil$ additional bits of memory per node.

Clearly, this trivial encoding mechanism stabilizes in $\kappa$ rounds. However, we can do much better. For simplicity, we do not try to give a tight bound here. 
Lemma 6.10. Happy nodes can communicate their counter values by sending only $O(1+B \log B)$ bits per $\kappa$ rounds, where $B=O(\log c / \log \kappa)$, in a way that stabilizes in $\kappa$ rounds.

Proof. First, we fix two unique bit strings HAPPY and UNHAPPY both having a length of $O(1)$ bits. We mark all messages from unhappy nodes with the header UNHAPPY. Happy nodes $v \in V \backslash \mathcal{F}$ send the bit string HAPPY in rounds $r$ when $a(v, r) \bmod \kappa=0$. In this and the subsequent $\kappa-1$ rounds, they furthermore send up to $b$ bits in order to encode the value of $a(v, r) \in[c]$, where they avoid the two excluded unique bit strings HAPPY and UNHAPPY. Since we are only interested in the asymptotic behavior, we may neglect these possible collisions and determine how large $b$ must be so that in $\kappa$ rounds we can encode $c$ different values.

Since there are $\kappa$ rounds in which to broadcast a message, we can think of each round as being a bin containing the bits broadcast by a node. Suppose we have $B=b / \log b$ uniquely labeled balls that we can place in $\kappa$ different bins. This way we can encode $B$-length strings over an alphabet of size $\kappa$ by interpreting each ball in a bin $i \in[\kappa]$ as giving the indices for the symbol $i$. This allows us to encode a total of $\kappa^{B}$ distinct values.

Since encoding the unique label of a single ball takes $O(\log B)$ bits and we can use constant-sized delimiters when encoding the set of balls in a single bin, we need $O(B \log B)$ bits to encode all the values. Thus, each node communicates a total of $O(B \log B)=O(b)$ bits during the course of $\kappa$ rounds. In order to encode $c$ different values, it suffices to satisfy $c \leq \kappa^{B}$. This can be done by choosing $B \geq \log c / \log \kappa$. Taking into account the bits for delimiters and the HAPPY string, the claim then follows.

Overall, we obtain the following theorem.

Theorem 6.11. For any integers $n>1, f<n / 3, \kappa=\Omega(f)$, and $c=\kappa j$ for $j>0$, there exists an $f$-resilient synchronous c-counter that runs on $n$ nodes, stabilizes in $O(\kappa)$ rounds, and requires $O\left(\log ^{2} f+\log c\right)$ bits to encode the state of a node. Moreover, once stabilized, nodes send only $O(1+B \log B)$ bits per $\kappa$ rounds, where $B=O(\log c / \log \kappa)$.

Proof. Let $\mathbf{A} \in \mathcal{A}(n, f, c)$ be an algorithm given by Theorem 1.1. As $T(\mathbf{A})=\Theta(f)$, for any $\kappa>T(\mathbf{A})$, the claim now directly follows from Corollaries 6.8 and 6.9 and Lemma 6.10 , where we note that only a constant number of variables of size at most $\max \{T(\mathbf{A}), c\}$ need to be encoded in the state of a node.

We remark that since $\kappa>T(\mathbf{A})=\Theta(f)$, in case of optimal resilience and $c=n^{O(1)}$, it holds that $B=O(1)$, and thus also, $O(1+B \log B)=O(1)$.

Corollary 6.12. For any $n>1$ and $c=n^{O(1)}$ that is an integer multiple of $n$, there exists a synchronous c-counter that runs on $n$ nodes, has optimal resilience $f=\lfloor(n-1) / 3\rfloor$, stabilizes in $\Theta(n)$ rounds, requires $O\left(\log ^{2} n\right)$ bits to encode the state of a node, and for which after stabilization correct nodes broadcast aysmptotically optimal $O(1)$ bits per $\Theta(n)$ rounds.

Proof. All properties except for the optimality of the last point follow from the choice of parameters by picking $\kappa=\Theta(n)$ in Theorem 6.11. The claimed optimality follows from the fact that in order to prove to a node that its counter value is inconsistent with that of others, it must receive messages from at least $f+1=\Theta(n)$ nodes; to guarantee stabilization in $O(n)$ rounds, this must happen every $\Omega(n)$ rounds for each correct node. 
7. Sending fewer messages. So far we have considered the size of messages nodes need to broadcast every round. In the case of the algorithm given in Theorem 1.1, every node will send $S=O\left(\log ^{2} f+\log c\right)$ bits in each round. As there are $\Theta\left(n^{2}\right)$ communication links, the total number of communicated bits in each round is $\Theta\left(S \cdot n^{2}\right)$. In this section, we consider a randomized variant of the algorithm that achieves better message and bit complexities in a slightly different communication model.

7.1. Pulling model. Throughout this section we consider the following variant of our communication model, where in every synchronous round $t$ each correct node $v$ :

1. contacts a subset $C(v, t) \subseteq V$ of other nodes to pull information from,

2. pulls a response message $r_{u} \in \mathcal{M}$ from every contacted node $u \in C(v, t)$,

3. updates its local state according to its current state and the responses it received.

Thus, every round $t$ node $v$ obtains a message vector $\mathbf{m}=\left\langle m_{0}, \ldots m_{n-1}\right\rangle$, where $m_{u}=r_{u}$ if $u \in C(v, t)$ and $m_{u}=\perp$, otherwise. Besides this modification, the model of computation is as before: Node $v$ updates its state using the state transition function $g:[n] \times X \times \mathcal{M}^{n} \rightarrow X$ and a correct node $u$ in state $x_{u}$ responds with the message $\mu\left(x_{u}\right)$, where $\mu: X \rightarrow \mathcal{M}$ maps the internal state of a node to a message. However, in the pulling model, the algorithm also needs to specify the set $C(v, t)$ of nodes it contacts every round. We assume that every correct node chooses this set randomly independent of its internal state.

As before, faulty nodes may respond with arbitrary messages that can be different for different pulling nodes. We define the (per-node) message and bit complexities of the algorithm as the maximum number of messages and bits, respectively, pulled by a nonfaulty node in any round.

This model is motivated by the challenges of designing energy-limited fault-tolerant circuits. We suggest the approach in which each node that makes a request for data also has to provide the energy resources for processing and answering the request. This way by limiting the energy supply of each individual node, we can also effectively limit the total amount of energy wasted due to the actions of the Byzantine nodes. However, to make this approach feasible, we have to design an algorithm in which each nonfaulty node needs to make only a few requests for data. In this section we design a randomized algorithm that satisfies this property.

7.2. High-level idea of the probabilistic construction. To keep the number of pulls, and thus number of messages sent, small, we modify the construction of Theorem 4.1 to use random sampling where useful. Essentially, the idea is to show that with high probability (w.h.p.) a small set of sampled messages accurately represents the current state of the system and the randomized algorithm will behave as the deterministic one. There are two steps where the nodes rely on information broadcast by the all the nodes: The majority voting scheme over the blocks and the variant of the phase king algorithm. In the following, both are shown to work under the sampling scheme with high probability by using concentration bound arguments.

More specifically, here w.h.p. means that for any constant $k \geq 1$ the probability of failure is bounded above by $\eta^{-k}$ when sampling $K=\Theta(\log \eta)$ messages (where the constants in the asymptotic notation may depend on $k$ ); here $\eta$ denotes the total number of nodes in the system after the recursive application of the resilience boosting procedure described in section 5. The idea is to use a union bound over all levels of recursion, nodes, and considered rounds to show that the sampling succeeds w.h.p. in all cases. For the randomized variant of Theorem 1.1, we will require the following additional constraint: When constructing a counter on $n$ nodes, the total number of 
failures is bounded by $f<\frac{n}{3+\gamma}$, where $\gamma>0$ is constant.

This allows us to construct probabilistic synchronous c-counters in the sense that we say that the counter stabilizes in time $T$, if for each round $t \geq T$ all nonfaulty nodes count correctly with probability $1-\eta^{-k}$.

7.3. Sampling communication channels. As discussed, there are two steps in the construction of Theorem 4.1 where we rely on broadcasting: (1) the majority voting scheme for electing a leader block and counter, and (2) the execution of the phase king protocol. For the sake of clarity, we only focus on modifying the basic algorithm, where the nodes broadcast their entire state each round. We start with a sampling lemma we use for both steps. First, recall the following concentration bound for the sum of independent random binary variables:

LEMma 7.1 (Chernoff's bound). Let $X=\sum X_{i}$ be a sum of independent random variables $X_{i} \in\{0,1\}$. Then for $0<\delta<1$,

$$
\operatorname{Pr}[X \leq(1-\delta) \mathbf{E}[X]] \leq \exp \left(-\frac{\delta^{2}}{2} \mathbf{E}[X]\right) .
$$

LEMma 7.2. Let $U \subseteq V$ be a nonempty set of nodes such that the fraction of faulty nodes in $U$ is strictly less than $1 /(3+\gamma)$. Suppose we sample $K$ nodes $v_{0}, \ldots, v_{K-1}$ uniformly at random from the set $U$. For a given local variable $x(\cdot, r)$ encoded in the nodes' local state on round $r \geq 0$ and a value $y$, define the random variable

$$
X_{i}= \begin{cases}1 & \text { if } x\left(v_{i}, r\right)=y \text { and } v_{i} \notin \mathcal{F}, \\ 0 & \text { otherwise }\end{cases}
$$

for each $i \in[K]$, and let $X=\sum_{i=0}^{K-1} X_{i}$ be the number of $y$ values sampled from correct nodes. There exists $K_{0}(\eta, k, \gamma)=\Theta(\log \eta)$ such that $K \geq K_{0}$ implies the following w.h.p.:

(a) If $x(u, r)=y$ for all $u \in U \backslash \mathcal{F}$, then $X \geq 2 K / 3$.

(b) If a majority of nodes $u \in U \backslash \mathcal{F}$ have $x(u, r)=y$, then $X \geq K / 3$.

(c) If $X \geq 2 K / 3$, then $|\{x(u, r)=y: u \in U \backslash \mathcal{F}\}| \geq|U \backslash \mathcal{F}| / 2$. in $U$.

Proof. Define $\delta=1-\frac{2}{3} \cdot \frac{3+\gamma}{2+\gamma}$ and let $\rho<1 /(3+\gamma)$ be the fraction of faulty nodes

(a) If all correct nodes $u \in U \backslash \mathcal{F}$ agree on value $x(u, r)=y$, then

$$
\mathbf{E}[X]=(1-\rho) K>\frac{2+\gamma}{3+\gamma} K .
$$

As $\delta$ satisfies $(1-\delta) \mathbf{E}[X]>2 K / 3$, it follows from Chernoff's bound that

$$
\begin{aligned}
\operatorname{Pr}\left[X<\frac{2 K}{3}\right] & \leq \operatorname{Pr}[X<(1-\delta) \mathbf{E}[X]] \\
& \leq \exp \left(-\frac{\delta^{2}}{2} \mathbf{E}[X]\right) \\
& \leq \exp \left(-\delta^{2} \frac{2+\gamma}{2(3+\gamma)} K\right) .
\end{aligned}
$$

If $K_{0}(\eta, k, \gamma)=\Theta(\log \eta)$ is sufficiently large, $K \geq K_{0}(\eta, k, \gamma)$ implies that this probability is bounded by $\eta^{-k}$. 
(b) If a majority of nonfaulty nodes $u$ have value $x(u, r)=y$, then

$$
\mathbf{E}[X] \geq \frac{1}{2}(1-\rho) K>\frac{1}{2} \cdot \frac{2+\gamma}{3+\gamma} K .
$$

As above, by picking the right constants and using concentration bounds, we get that

$$
\begin{aligned}
\operatorname{Pr}\left[X \leq \frac{K}{3}\right] & \leq \operatorname{Pr}[X<(1-\delta) \mathbf{E}[X]] \\
& \leq \exp \left(-\frac{\delta^{2}}{2} \mathbf{E}[X]\right) \\
& \leq \exp \left(-\delta^{2} \frac{2+\gamma}{4(3+\gamma)} K_{0}\right) \leq \eta^{-k}
\end{aligned}
$$

(c) Suppose the majority of correct nodes have values different from $y$. Define

$$
\bar{X}_{i}= \begin{cases}1 & \text { if } x\left(v_{i}, r\right) \neq y \text { and } v_{i} \notin \mathcal{F}, \\ 0 & \text { otherwise }\end{cases}
$$

and $\bar{X}=\sum_{i=0}^{K-1} \bar{X}_{i}$ as the random variable counting the number of samples with values different from $y$. Arguing as for (b), we see that

$$
\operatorname{Pr}\left[X \geq \frac{2 K}{3}\right]=\operatorname{Pr}\left[\bar{X}<\frac{K}{3}\right] \leq \eta^{-k}
$$

where again we assume that $K_{0}(\eta, k, \gamma)=\Theta(\log \eta)$ is sufficiently large. Thus, $X$ $\geq 2 K / 3$ implies w.h.p. that the majority of correct nodes have value $y$.

Randomized majority voting. Recall that in the majority voting scheme, there are four local variables, two for each $i \in\{0,1\}$, whose values depend directly on the messages broadcast by all nodes:

- $m_{i}(v, r)$ stores the most frequent counter value in block $i$ in round $r$, which is determined from the broadcast output variables of $\mathbf{A}_{i}$ with ties broken arbitrarily, and

- $M_{i}(v, r)$ stores the majority vote on $m_{i}(v, r-1)$.

Throughout the remainder of this section, we let $K=\Theta(\log \eta)$ such that $K \geq K_{0}$ as given by Lemma 7.2. Let $m_{i}^{*}(v, r)$ be the sampled version of $m_{i}(v, r)$; here the value is determined by taking a random sample of size $K$ from the set $V_{i}$. Analogously, the variable $M_{i}^{*}(v, r)$ is determined by taking a random sample of size $K$ from the set $V$ and taking the value that appears at least $2 K / 3$ times in the sample.

Remark 7.3. It holds that $f_{i} / n_{i}<1 /(3+\gamma)$ for $i \in\{0,1\}$.

Lemma 7.4. Suppose block $i \in\{0,1\}$ is correct. Then for all $v \in V \backslash \mathcal{F}$ and $r \geq T\left(\mathbf{A}_{i}\right)$, we have

$$
\begin{aligned}
m_{i}^{*}(v, r) & =m_{i}(v, r), \\
M_{i}^{*}(v, r+1) & =M_{i}(v, r+1)
\end{aligned}
$$

w.h.p.

Proof. To show the claim, we will apply Lemma 7.2 with $U=V$ and $U=V_{i}$. Before this, note that the fraction of faulty nodes in both $V$ and $V_{i}$ is less than $1 /(3+\gamma)$ : 
By assumption, we have $f / n<1 /(3+\gamma)$ and by Remark 7.3 yield $f_{i} / n_{i}<1 /(3+\gamma)$. Thus, in both cases, we satisfy the first condition of Lemma 7.2.

For the claim regarding variable $m_{i}$, we apply Lemma 7.2 with $U=V_{i}$, that is, sample the subset $V_{i} \subseteq V$ consisting of nodes in block $i$. Since $\left|V_{i}\right|=n_{i}$ and $i$ is a correct block, the set $V_{i}$ contains at most $f_{i}$ faulty nodes and all correct nodes output the same value $y \in\left[c_{i}\right]$, as $\mathbf{A}_{i}$ has stabilized by round $r \geq T\left(\mathbf{A}_{i}\right)$. Moreover, $f_{i} / n_{i}<\frac{1}{3+\gamma}$ by Remark 7.3, so statement (a) of Lemma 7.2 yields that w.h.p. at least a fraction of $2 / 3$ of the sampled nodes output $y$.

To show the claim for variable $M_{i}^{*}$, note that by the previous case, $m_{i}^{*}(v, r)=$ $m_{i}(v, r)$ holds for all correct nodes $v$ w.h.p. Applying statement (a) of Lemma 7.2 to the set $V$ and variable $m_{i}^{*}(v, r)$, we get that at least a fraction of $2 / 3$ of the samples have the same value.

From Lemma 7.4 it follows that we get probabilistic - in the sense that the claims hold w.h.p.- - variants of Lemmas 4.4-4.6. These, in turn, yield the following probabilistic variant of Corollary 4.7.

Conollany 7.5. There is a round $r=T+O(f)$ so that for all $v, w \in V \backslash \mathcal{F}$ w.h.p. it holds that

1. $d(v, r)=d(w, r)$ and

2. for all $r^{\prime} \in\{r+1, \ldots, r+\tau-1\}$ we have $d\left(v, r^{\prime}\right)=d\left(v, r^{\prime}-1\right)+1 \bmod \tau$.

Randomized phase king. To obtain a randomized variant of the phase king algorithm, we modify the threshold votes used in the algorithm as follows. Instead of checking whether at least $n-f$ of all messages have the same value, we check whether at least a fraction of $2 / 3$ of the sampled messages have the same value. Similarly, when checking for at least $f+1$ values, we check whether a fraction of $1 / 3$ of the sampled messages have this value.

As a corollary, we get that when using the sampling scheme in the pulling model, the execution of the phase king essentially behaves as in the deterministic broadcast model.

COROLLARY 7.6. When executing the randomized variant of the phase king protocol from section 4 for $\eta^{O(1)}$ rounds, the statements of Lemmas 4.8 and 4.9 hold w.h.p.

Proof. The modified phase king algorithm given in subsection 4.3 uses two thresholds, $n-f$ and $f+1$. As discussed, these are replaced with threshold values of $2 K / 3$ and $K / 3$ when taking $K \geq K_{0}(\eta, k, \gamma)$ samples. Using the statements of Lemma 7.2, we can argue analogously to the proofs of Lemmas 4.8 and 4.9 .

First, to see that Lemma 4.8 holds w.h.p., note that from statements (b) and (c) of Lemma 7.2, it follows that if a node samples $2 K / 3$ times value $y$, then w.h.p. other nodes sample at least $K / 3$ times the same value (that is, we get the probabilistic version of Lemma 4.3). Now we can follow the same reasoning as in Lemma 4.8.

Similarly, it is straightforward to check that Lemma 4.9 holds w.h.p.: If all correct nodes agree on $a(\cdot)$, then all correct nodes sample at least $2 K / 3$ times the same value w.h.p. by statement (a) of Lemma 7.2. Thus, analogously as in the proof of Lemma 4.9, we get that the agreement persists when executing $I_{3 k}, I_{3 k+1}$, or $I_{3 k+2}$ w.h.p.

Finally, we can apply the union bound over all $\eta^{O(1)}$ rounds and samples taken by correct nodes $\left(n-f \leq \eta\right.$ per round), that is, in total over $\eta^{O(1)}$ events. By choosing large enough $k=O(1)$, we get that the claim holds w.h.p. $1-\eta^{-k}$.

7.4. Randomized resilience boosting. It remains to formulate the probabilistic variant of Theorem 4.1. To this end, define $\mathcal{P}(n, f, c, \eta, k)$ as the family of 
probabilistic synchronous $c$-counters on $n$ nodes of resilience $f$. Here, probabilistic means that an algorithm $\mathbf{P} \in \mathcal{P}(n, f, c, \eta, k)$ with stabilization time $T(\mathbf{P})$ merely guarantees that it counts correctly with probability $1-\eta^{-k}$ in any given round $t \geq T(\mathbf{P})$.

Let $P(\mathbf{P})$ denote the number of messages pulled per node by a probabilistic counter $\mathbf{P} \in \mathcal{P}(n, f, c, \eta, k)$. For any deterministic algorithm $\mathbf{A} \in \mathcal{A}(n, f, c)$, we define $P(\mathbf{A})=n$.

Theorem 7.7. Let $c, n>1$ and $f<n /(3+\gamma)$, where $\gamma>0$ and $n \leq \eta$. Define $n_{0}=\lfloor n / 2\rfloor, n_{1}=\lceil n / 2\rceil, f_{0}=\lfloor(f-1) / 2\rfloor, f_{1}=\lceil(f-1) / 2\rceil$, and $\tau=3(f+2)$. If for $i \in\{0,1\}$ there exist synchronous counters $\mathbf{A}_{i} \in \mathcal{A}\left(n_{i}, f_{i}, c_{i}\right)$ such that $c_{i}=3^{i} \cdot 2 \tau$, then for any sufficiently large $k=O(1)$, there exists a probabilistic synchronous c-counter $\mathbf{B} \in \mathcal{P}(n, f, c, \eta, k)$ that

- stabilizes in $T(\mathbf{B})=\max \left\{T\left(\mathbf{A}_{0}\right), T\left(\mathbf{A}_{1}\right)\right\}+O(f)$ rounds,

- has state complexity of $S(\mathbf{B})=\max \left\{S\left(\mathbf{A}_{0}\right), S\left(\mathbf{A}_{1}\right)\right\}+O(\log f+\log c)$ bits, and

- each node pulls at most $P(\mathbf{B})=\max \left\{P\left(\mathbf{A}_{0}\right), P\left(\mathbf{A}_{1}\right)\right\}+O(\log \eta)$ messages per round.

Proof. The proof proceeds analogously to the proof of Theorem 4.1. First, we apply Corollary 7.5 to get a round counter that works once in a while w.h.p. We can then use this to clock the randomized phase king and Corollary 7.6 implies that the new output counter will reach agreement in $O(f)$ rounds w.h.p. The time and state complexities are as in the proof of Theorem 4.1.

To analyze the number of pulls, observe that in Lemma 7.4 each node samples twice $K=O(\log \eta)$ messages (from both $V_{0}$ and $\left.V_{1}\right)$ and Corollary 7.6 samples $O(\log \eta)$ messages from all the nodes. Thus, in total, a node $v \in V_{i}$ samples $O(\log \eta)$ messages in addition to the messages pulled when executing $\mathbf{A}_{i}$.

Note that we can choose to replace $\mathbf{A} \in \mathcal{A}(n, f, c)$ by $\mathbf{Q} \in \mathcal{P}(n, f, c, \eta, k)$ when applying this theorem, arguing that w.h.p. it behaves like a corresponding algorithm $\mathbf{A} \in \mathcal{A}(n, f, c)$ for polynomially many rounds. Furthermore, note that it is also possible to boost the probability of success, and thus the period of stability, by simply increasing the sample size. For instance, sampling polylog $\eta$ messages yields an error probability of $\eta^{- \text {polylog } \eta}$ in each round, whereas in the extreme case, by "sampling" all nodes the algorithm reduces to the deterministic case.

Using Theorem 7.7 recursively as in section 5 for $O(\log f)$ steps, we get the following result.

TheOREM 7.8. For any integers $c, n>1, f<n /(3+\gamma)$, there exists an $f$-resilient probabilistic synchronous c-counter that runs on n nodes, requires $O\left(\log ^{2} f+\log c\right)$ bits to encode the state of a node, has each node pull $O(\log f \log n)$ messages per round, and stabilizes in $O(f)$ rounds with probability $1-n^{-k}$, where $k>0$ is a freely chosen constant.

7.5. Oblivious adversary. Finally, we remark that under an oblivious adversary, that is, an adversary that picks the set of faulty nodes independently of the randomness used by the nonfaulty nodes, we get pseudorandom synchronous counters satisfying the following: (1) the execution stabilizes w.h.p. and (2) if the execution stabilizes, then all nonfaulty nodes will deterministically count correctly. In other words, we can fix the random bits used by the nodes to sample the communication links once, and w.h.p. we sample sufficiently many communication links to nonfaulty nodes for the algorithm to (deterministically) stabilize. This gives us the following result. 
Corollary 7.9. For any integers $c, n>1, f<n /(3+\gamma)$, there exists a pseudorandom synchronous c-counter with resilience $f$ against an oblivious fault pattern that runs on $n$ nodes, requires $O\left(\log ^{2} f+\log c\right)$ bits to encode the state of a node, has each node pull $O(\log f \log n)$ messages per round, and stabilizes in $O(f)$ rounds.

8. Conclusions. In this work, we showed that there exist algorithms for synchronous counting that (1) are deterministic, (2) tolerate the optimal number of faults, (3) have asymptotically optimal stabilization time, and (4) need to store and communicate a very small number of bits between consecutive rounds - something no prior algorithms have been able to do.

In addition, we discussed two complementary approaches on how to further reduce the total number of communicated bits in the network. The first one is a deterministic construction that lets the nodes communicate only a few bits after stabilization, in order to verify that stabilization has occurred and that the counters agree. The construction retains all properties (1)-(4), and in particular, when constructing polynomially sized counters with linear resilience, the algorithm communicates an asymptotically optimal number of bits after stabilization.

The second technique for reducing the amount of communication is based on random sampling of communication channels. Here, we employed randomization so that each node needs to communicate only with polylog $n$ instead of $n-1$ other nodes in the system, thus reducing the number of messages sent from $\Theta\left(n^{2}\right)$ to $\Theta(n$ polylog $n)$. The trade-off here is that the resulting algorithm has slightly suboptimal resilience of $f<n /(3+\gamma)$, where $\gamma>0$ is a constant, and is merely guaranteed to work for polynomially many rounds w.h.p. before a new stabilization phase is required. The latter issue disappears when employing pseudorandomness. In this case, one may simply fix a random topology and the algorithm will not fail again after stabilization; naturally, this necessitates that the Byzantine faulty nodes are chosen in an oblivious manner, i.e., independently of the topology.

We can also combine both techniques to attain probabilistic counters that during stabilization communicate $\Theta(n$ polylog $n)$ bits each round and after stabilization asymptotically optimal $O(1)$ bits every $\Theta(n)$ rounds.

To conclude the paper, we now wish to highlight some interesting problems that still remain open:

Q1. Our solutions are not adaptive (as defined in [23]), as their stabilization time is not bounded by a function of the number of actual permanent faults. Can this be achieved?

Q2. Are there algorithms that satisfy (1)-(3), but need to store and communicate substantially fewer than $\log ^{2} f$ bits? This question has been partially answered in follow-up work [25], showing that $O(\log f)$ bits suffice. However, no nontrivial lower bound is known, so it remains open whether $o(\log f)$ bits suffice.

Q3. Can the ideas presented in this paper be applied to randomized consensus routines in order to achieve sublinear stabilization time with high resilience and small communication overhead? Again, a partial answer is provided in [25]: this is possible, but the given solutions may still fail after stabilization (with a very small probability per round). The question thus remains open w.r.t. the original problem definition, which requires that after stabilization the algorithm keeps counting correctly.

Finally, we point out that the recursive approach we employ in this paper can be interpreted as an extension of its similar use in synchronous consensus routines [5, 6], 
where the shared round counter is implicitly given by the synchronous start.

Q4. Can a similar recursive approach also be used for deriving improved pulse synchronization $[14,18]$ algorithms?

Interestingly, no reduction from consensus to pulse synchronization is known, so there is still hope for efficient deterministic pulse synchronization algorithms that stabilize in sublinear time.

Acknowledgment. We thank the anonymous reviewers for their helpful comments.

\section{REFERENCES}

[1] M. Ajtai And N. Linial, The influence of large coalitions, Combinatorica, 13 (1993), pp. 129145, https://doi.org/10.1007/BF01303199.

[2] A. Arora, S. Dolev, and M. G. Gouda, Maintaining digital clocks in step, Parallel Process. Lett., 1 (1991), pp. 11-18, https://doi.org/10.1142/S0129626491000161.

[3] B. Awerbuch, S. Kutten, Y. Mansour, B. Patt-Shamir, and G. Varghese, A time-optimal self-stabilizing synchronizer using a phase clock, IEEE Trans. Dependable Secure Comput., 4 (2007), pp. 180-190.

[4] M. Ben-Or, D. Dolev, And E. N. Hoch, Fast self-stabilizing Byzantine tolerant digital clock synchronization, in Proceedings of the 27th Annual ACM Symposium on Principles of Distributed Computing (PODC 2008), ACM, New York, 2008, pp. 385-394, https: //doi.org/10.1145/1400751.1400802.

[5] P. Berman, J. A. Garay, and K. J. Perry, Bit optimal distributed consensus, in Computer Science: Research and Applications, Springer, Boston, pp. 313-321, https://doi.org/10. 1007/978-1-4615-3422-8_27.

[6] P. Berman, J. A. Garay, and K. J. Perry, Towards optimal distributed consensus, in Proceedings of the 30th Annual Symposium on Foundations of Computer Science (FOCS 1989), IEEE, Washington, DC, 1989, pp. 410-415, https://doi.org/10.1109/SFCS.1989. 63511.

[7] L. Boczkowski, A. Korman, and E. Natale, Minimizing message size in stochastic communication patterns: Fast self-stabilizing protocols with 3 bits, in Proceedings of the 28th Annual ACM-SIAM Symposium on Discrete Algorithms (SODA 2017), SIAM, Philadelphia, 2017, pp. 2540-2559, https://doi.org/10.1137/1.9781611974782.168.

[8] C. Boulinier, F. Petit, AND V. Villain, Synchronous vs. asynchronous unison, Algorithmica, 51 (2008), pp. 61-80, https://doi.org/10.1007/s00453-007-9066-x.

[9] C. Delporte-Gallet, S. Devismes, and H. Fauconnier, Robust stabilizing leader election, in Proceedings of the 9th Symposium on Stabilization, Safety, and Security of Distributed Systems, Springer-Verlag, Berlin, Heidelberg, 2007, pp. 219-233.

[10] S. Devismes, T. Masuzawa, and S. Tixeuil, Communication efficiency in self-stabilizing silent protocols, in Proceedings of the 29th Conference on Distributed Computing Systems (ICDCS), IEEE Computer Society Washington, DC, 2009, pp. 474-481, https://doi.org/10. 1109/ICDCS.2009.24.

[11] D. Dolev, The Byzantine generals strike again, J. Algorithms, 3 (1982), pp. 14-30.

[12] D. Dolev, M. FügGer, C. Lenzen, U. Schmid, And A. Steininger, Fault-tolerant distributed systems in hardware, Bulletin of the EATCS, 116 (2015), http://bulletin.eatcs.org/index. $\mathrm{php} /$ beatcs/issue/view/18.

[13] D. Dolev, K. Heljanko, M. Järvisalo, J. H. Korhonen, C. Lenzen, J. Rybicki, J. Suomela, AND S. WIERINGA, Synchronous counting and computational algorithm design, J. Comput. System Sci., 82 (2016), pp. 310-332, https://doi.org/10.1016/j.jcss.2015.09.002.

[14] D. Dolev AND E. N. Hoch, On self-stabilizing synchronous actions despite Byzantine attacks, in Proceedings of the 21st International Symposium on Distributed Computing (DISC 2007), Lecture Notes in Comput. Sci. 4731, Springer, Berlin, Heidelberg, 2007, pp. 193-207, https://doi.org/10.1007/978-3-540-75142-7_17.

[15] D. Dolev, J. H. Korhonen, C. Lenzen, J. Rybicki, And J. Suomela, Synchronous counting and computational algorithm design, in Proceedings of the 15th International Symposium on Stabilization, Safety, and Security of Distributed Systems (SSS 2013), Lecture Notes in Comput. Sci. 8255, Springer-Verlag, New York, 2013, pp. 237-250, https://doi.org/10.1007/ 978-3-319-03089-0_17. 
[16] D. Dolev And R. Reischuk, Bounds on information exchange for Byzantine agreement, J. ACM, 32 (1985), pp. 191-204, https://doi.org/10.1145/2455.214112.

[17] S. Dolev, Self-Stabilization, The MIT Press, Cambridge, MA, 2000.

[18] S. Dolev And J. L. Welch, Self-stabilizing clock synchronization in the presence of Byzantine faults, J. ACM, 51 (2004), pp. 780-799, https://doi.org/10.1145/1017460.1017463.

[19] S. Dubois, M. Potop-Butucaru, M. Nesterenko, and S. Tixeull, Self-stabilizing byzantine asynchronous unison, J. Parallel Distrib. Comput., 72 (2012), pp. 917-923, https://doi.org/ 10.1016/j.jpdc.2012.04.001.

[20] M. J. Fischer And N. A. Lynch, A lower bound for the time to assure interactive consistency, Inform. Process. Lett., 14 (1982), pp. 183-186, https://doi.org/10.1016/0020-0190(82) 90033-3.

[21] M. G. Gouda And T. Herman, Stabilizing unison, Inform. Process. Lett., 35 (1990), pp. 171-175.

[22] E. Hoch, D. Dolev, And A. Daliot, Self-stabilizing Byzantine digital clock synchronization, in Proceedings of the 8th International Symposium on Stabilization, Safety, and Security of Distributed Systems (SSS 2006), Lecture Notes in Comput. Sci. 4280, Springer, Berlin, Heidelberg, 2006, pp. 350-362, https://doi.org/10.1007/978-3-540-49823-0_25.

[23] S. Kutten And B. PatT-Shamir, Adaptive stabilization of reactive protocols, in Proceedings of the 24th Conference on Foundations of Software Technology and Theoretical Computer Science (FSTTCS), Springer-Verlag, Berlin, Heidelberg, 2005, pp. 396-407, https://doi.org/ 10.1007/978-3-540-30538-5_33.

[24] C. Lenzen And J. RyBicki, Efficient counting with optimal resilience, in Proceedings of the 29th International Symposium on Distributed Computing (DISC 2015), Lecture Notes in Comput. Sci. 9363, Springer, Berlin, Heidelberg, 2015, pp. 16-30, https://doi.org/10.1007/ 978-3-662-48653-5_2.

[25] C. Lenzen and J. RyBicki, Near-optimal self-stabilising counting and firing squads, in Proceedings of the 18th Symposium on Stabilization, Safety, and Security of Distributed Systems (SSS), Lecture Notes in Comput. Sci. 10083, Springer, Cham, 2016, pp. 263-280, https://doi.org/10.1007/978-3-319-49259-9_21.

[26] C. Lenzen, J. Rybicki, And J. Suomela, Towards optimal synchronous counting, in Proceedings of the 34th Annual ACM Symposium on Principles of Distributed Computing (PODC 2015), ACM, New York, 2015, pp. 441-450, https://doi.org/10.1145/2767386.2767423.

[27] M. C. Pease, R. E. Shostak, and L. Lamport, Reaching agreement in the presence of faults, J. ACM, 27 (1980), pp. 228-234, https://doi.org/10.1145/322186.322188.

[28] T. Takimoto, F. Ooshita, H. Kakugawa, and T. Masuzawa, Communication-efficient selfstabilization in wireless networks, in Proceedings of the 14th Conference on Stabilization, Safety, and Security of Distributed Systems (SSS), Lecture Notes in Comput. Sci. 7596, Springer, Berlin, Heidelberg, 2012, pp. 1-15, https://doi.org/10.1007/978-3-642-33536-5_1. 